\title{
Semaphorin 3F Is a Bifunctional Guidance Cue for Dopaminergic Axons and Controls Their Fasciculation, Channeling, Rostral Growth, and Intracortical Targeting
}

\author{
Sharon M. Kolk, ${ }^{1}$ Rou-Afza F. Gunput, ${ }^{1}$ Tracy S. Tran, ${ }^{2}$ Dianne M. A. van den Heuvel, ${ }^{1}$ Asheeta A. Prasad, ${ }^{1}$ \\ Anita J. C. G. M. Hellemons, ${ }^{1}$ Youri Adolfs, ${ }^{1}$ David D. Ginty, ${ }^{2}$ Alex L. Kolodkin, ${ }^{2}$ J. Peter H. Burbach, ${ }^{1}$ Marten P. Smidt, ${ }^{1}$ \\ and R. Jeroen Pasterkamp ${ }^{1}$ \\ ${ }^{1}$ Department of Neuroscience and Pharmacology, Rudolf Magnus Institute of Neuroscience, University Medical Center Utrecht, 3584 CG Utrecht, The \\ Netherlands, and ${ }^{2}$ The Solomon H. Snyder Department of Neuroscience and Howard Hughes Medical Institute, The Johns Hopkins University School of \\ Medicine, Baltimore, Maryland 21205
}

Dopaminergic neurons in the mesodiencephalon (mdDA neurons) make precise synaptic connections with targets in the forebrain via the mesostriatal, mesolimbic, and mesoprefrontal pathways. Because of the functional importance of these remarkably complex ascending axon pathways and their implication in human disease, the mechanisms underlying the development of these connections are of considerable interest. Despite extensive in vitro studies, the molecular determinants that ensure the perfect formation of these pathways in vivo remain mostly unknown. Here, we determine the embryonic origin and ontogeny of the mouse mesoprefrontal pathway and use these data to reveal an unexpected requirement for semaphorin $3 \mathrm{~F}$ (Sema3F) and its receptor neuropilin-2 (Npn-2) during mdDA pathway

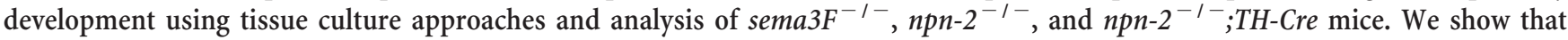
Sema3F is a bifunctional guidance cue for mdDA axons, some of which have the remarkable ability to regulate their responsiveness to Sema3F as they develop. During early developmental stages, Sema3F chemorepulsion controls previously uncharacterized aspects of mdDA pathway development through both Npn-2-dependent (axon fasciculation and channeling) and Npn-2-independent (rostral growth) mechanisms. Later on, chemoattraction mediated by Sema3F and Npn-2 is required to orient mdDA axon projections in the cortical plate of the medial prefrontal cortex. This latter finding demonstrates that regulation of axon orientation in the target field occurs by chemoattractive mechanisms, and this is likely to also apply to other neural systems. In all, this study provides a framework for additional dissection of the molecular basis of mdDA pathway development and disease.

\section{Introduction}

The formation of long ascending and descending fiber systems in the CNS is exquisitely complex and requires a myriad of extracel-

\footnotetext{
Received May 31, 2009; revised Aug. 10, 2009; accepted Sept. 1, 2009.
}

This work was supported by grants from The Netherlands Organization for Scientific Research and the Dutch Brain Foundation [HsN H04.10, VENI 916.56.104 (S.M.K.); HsN 13F05(2).27, VENI 863.04.001, VIDI 917.76.357 (R.J.P.)], the International Parkinson Foundation, the Division of Neuroscience of University Medical Center Utrecht, and the Genomics Center Utrecht (R.J.P.). R.J.P. is a recipient of a Human Frontier Science Program Organization Career Development Award and was a Henry and William Test National Alliance for Research on Schizophrenia and Depression Investigator. D.D.G. and A.L.K. are investigators of the Howard Hughes Medical Institute. We thank Stephan Lammel for critically reading this manuscript and members of the Pasterkamp, Kolodkin, and Ginty Laboratories for helpful discussions and technical advice. We are grateful to Dr. P. Mombaerts (Max Planck Institute for Biophysics, Frankfurt am Main, Germany) and Dr. C. R. Gerfen (National Institutes of Health, Bethesda, MD) for providing us with $n p n-2$ conditional mice and $T H$-Cre transgenic mice, respectively. We thank Dr. J. Verhaagen (Netherlands Institute for Neuroscience, Amsterdam, The Netherlands) for Sema3A cDNA, Dr. A. W. Püschel (Westfalische WilhelmsUniversität Münster, Münster, Germany) for Sema3C and Sema3F cDNAs, and Dr. R. F. Hevner (University of Washington, Seattle, WA) for the Tbr1 antibody. We thank Corlinda ten Brink for help with confocal microscopy.

Correspondence should be addressed to either Sharon M. Kolk at her present address or R. Jeroen Pasterkamp, Department of Neuroscience and Pharmacology, Rudolf Magnus Institute of Neuroscience, University Medical Center Utrecht, Universiteitsweg 100, 3584 CG Utrecht, The Netherlands, E-mail: S.Kolk@ncmls.ru.nl or r.j.pasterkamp@umcutrecht.nl.

S.M. Kolk's present address: Department of Molecular Animal Physiology, Donders Centre for Neuroscience, Nijmegen Centre for Molecular Life Sciences (NCMLS), Radboud University, Nijmegen, The Netherlands.

DOI:10.1523/JNEUROSCI.2521-09.2009

Copyright $\odot 2009$ Society for Neuroscience 0270-6474/09/2912542-16\$15.00/0 lular signals. Although the wiring of ascending and descending spinal pathways has been studied in detail, the mechanisms by which long ascending and descending axon tracts within the forebrain are established are poorly understood (Bagri et al., 2002; Lyuksyutova et al., 2003; Bourikas et al., 2005; Liu et al., 2005; Chauvet et al., 2007). Among the most prominent ascending fiber systems in the forebrain are the axon pathways from mesodiencephalic dopamine ( $\mathrm{mdDA}$ ) neurons in the substantia nigra (SN) and ventral tegmental area (VTA). mdDA neurons in the adult SN send projections to the dorsolateral striatum (the mesostrial pathway) and control voluntary movement. mdDA neurons in the VTA target the ventromedial striatum and medial prefrontal cortex (mPFC), forming the mesolimbic and mesoprefrontal pathways, respectively, and mediate cognitive functions (Björklund and Dunnett, 2007; Ikemoto, 2007; Lammel et al., 2008). Changes in mdDA connectivity cause drastic behavioral deficits and underlie neural disorders such as Parkinson's disease and schizophrenia (Winterer and Weinberger, 2004; Savitt et al., 2006), highlighting the need to understand how these precise connections are established and maintained.

The molecular events that orchestrate mdDA pathway development are just beginning to be understood (Van den Heuvel 
and Pasterkamp, 2008). In vitro studies reveal chemotactic activities demarcating the presumptive mdDA trajectory (Nakamura et al., 2000; Gates et al., 2004) and several axon guidance molecules can elicit mdDA guidance responses in vitro (Yue et al., 1999; Lin et al., 2005; Hernández-Montiel et al., 2008; Cooper et al., 2009; Yamauchi et al., 2009). On the basis of these studies, it seems conceivable that mdDA pathway development relies on the combinatorial actions of a multitude of molecular cues. Thus far, however, only few in vivo data have been reported. Analysis of Slit mutant mice implicates Slit-mediated repulsion in medial forebrain bundle (MFB) patterning (Bagri et al., 2002). In addition, genetic manipulation of EphA5 and ephrinA5 leads to a functional reduction in mdDA connections, whereas neuropilin-2 (npn-2)-deficient mice display rostrocaudal polarity defects (Halladay et al., 2004; Sieber et al., 2004; Cooper et al., 2009; Yamauchi et al., 2009). Despite this recent progress, the molecular events that underlie mdDA pathway development in vivo remain poorly understood. For example, molecules controlling mdDA axon fasciculation, longitudinal growth or pathwayspecific events have yet to be determined.

Here, we determine the embryonic origin and ontogeny of the mouse mesoprefrontal pathway and use these data to show an in vivo requirement for semaphorin $3 \mathrm{~F}$ (Sema3F) and its receptor Npn-2 during mesoprefrontal pathway development. Furthermore, our results identify Sema3F as a bifunctional guidance cue and show that some mdDA axons have the remarkable ability to change their Sema3F responsiveness as they develop. Finally, we find that Sema3F-Npn-2mediated axon attraction is necessary for the correct orientation of mdDA axons within the mPFC. This finding highlights a novel axonal wiring principle that may also apply to other neural systems.

\section{Materials and Methods}

Animals and tissue treatment. All animal use and care were in accordance with institutional guidelines. C57BL/6 mice were obtained from Charles River. Sema3F and $n p n-2$ mutant mice have been described previously (Giger et al., 2000; Sahay et al., 2003). Tyrosine hydroxylase (TH)-Cre mice were obtained from Dr. C. R. Gerfen (National Institutes of Health, Bethesda, MD) (Gong et al., 2007), and $n p n-2$ conditional mice were obtained from Dr. P. Mombaerts (Max Planck Institute of Biophysics, Frankfurt, Germany) (Walz et al., 2002). Timed-pregnant mice and pups were killed by means of cervical dislocation or decapitation, respectively. The morning on which a vaginal plug was detected was considered embryonic day 0.5 (E0.5), and the day of birth, postnatal day 0 (P0). For in situ hybridization experiments, E14.5 and E18.5 brains were directly frozen and sections were cut on a cryostat. For immunohistochemistry, embryos (E10.5-E13.5) and brains (E14.5-E18.5) were collected in PBS and fixed by immersion for $0.5-3 \mathrm{~h}$ in $4 \%$ paraformaldehyde (PFA) in $\mathrm{PBS}$ at $4^{\circ} \mathrm{C}$. Adult mice were transcardially perfused with ice-cold saline followed by $4 \%$ PFA and postfixed for $2 \mathrm{~h}$. Samples intended for immunohistochemistry were washed in PBS and cryoprotected in 30\% sucrose. Brains were frozen in M-1 embedding matrix (Thermo Fisher Scientific) on dry ice and stored at $-80^{\circ} \mathrm{C}$. Cryostat sections were cut at $14-16 \mu \mathrm{m}$, mounted on Superfrost Plus slides (Thermo Fisher Scientific), air-dried, and stored desiccated at $-20^{\circ} \mathrm{C}$.

Immunohistochemistry. Cryosections were stained immunohistochemically as described previously (Kolk et al., 2006). Sections were counterstained with either fluorescent Nissl stain (NeuroTrace; Invitrogen; 1:500) or propidium iodide (PI) (Invitrogen; 1:5000), washed extensively in PBS, and embedded in 90\% glycerol. Staining was visualized using epifluorescent illumination on a Zeiss Axioskop 2 microscope or by confocal laser-scanning microscopy (Zeiss LSM510). The primary antibodies used, their suppliers, and dilutions can be found in Table 1. The nomenclature to describe TH-positive cells and axons within different brain areas is as described previously by Schambra et al. (1992) and Jacobowitz and Abott (1998) and extended as outlined in Figure 3.
Table 1. Primary antibodies, their suppliers, and dilutions

\begin{tabular}{lll}
\hline Antibody & Supplier & Dilution \\
\hline Rabbit anti-TH & Pel-Freez & $1: 1000$ \\
Sheep anti-TH & Pel-Freez & $1: 200$ \\
Mouse anti- $\beta$ III-tubulin (Tuj1) & Covance & $1: 500$ \\
Rabbit anti-Tbr1 & A kind gift from Dr. R. F. Hevner (University of & $1: 1000$ \\
& $\quad$ Washington, Seattle, WA) & \\
Mouse anti-calbindin & Santa Cruz & $1: 100$ \\
Rabbit anti-Npn1 and rabbit & Swant & $1: 5000$ \\
$\quad$ anti-Npn-2 & Kolodkin et al. (1997); Giger et al. (1998) & $1: 50$ \\
Rabbit anti-GIRK2 & Alomone Labs & $1: 80$ \\
\hline
\end{tabular}

To assess the width of the MFB in wild-type (wt) mice and in sema $3 F^{-1-}, n p n-2^{-1-}$, and $n p n-2^{-1-} ; \mathrm{TH}$-Cre mutant mice and littermate controls, images were captured of sagittal cryosections through the telencephalon of E18.5 mouse embryos immunostained for TH or of sagittal vibratome sections of E18.5 embryos traced with DiI in the medial VTA (mVTA) system and counterstained with fluorescent Nissl. Subsequently, axon tract width was determined as described by Pasterkamp et al. (2003) using OpenLab software (Improvision). In brief, in each animal three sagittal sections were selected from each ipsilateral MFB and the MFB region was imaged from each section. One section always covered the central aspect of the MFB and was flanked by two other sections on either side within the MFB (spaced 40-100 $\mu \mathrm{m}$ apart). In each image, a line was drawn perpendicular to the direction of axon extension within the MFB at the location indicated in Figure $8 B$. The average of the six lines was designated as the width of the TH-positive MFB per embryo. To determine the number of thick axon fascicles in the MFB, a similar approach was taken. First, the mean width of a THpositive axon fascicle in the MFB of wild-type mice was determined by measuring the width of 150 axon fascicles in the MFB in TH-immunostained sagittal sections derived from three different wild-type mice. This mean width was established at $19.2 \mu \mathrm{m}$ (a similar width was found in DiIlabeled mice) (data not shown). This number was then used to determine how many axon fascicles $\geq 19.2 \mu \mathrm{m}$ crossed the lines used to establish the width of the MFB as outlined above. Data were tested for significance by one-way ANOVA $(\alpha=5 \%)$ and expressed as means \pm SEM. No statistical differences could be detected between wild-type mice and the different littermate controls with respect to MFB width and axon fascicle number.

For assessing TH axon density in the mPFC of wild-type mice and of sema $3 F^{-1-}, n p n-2^{-1-}$, and $n p n-2^{-1-} ;$ TH-Cre mutant mice and their littermate controls, three to five embryos were analyzed per developmental stage and two to four well spaced $(80 \mu \mathrm{m})$ sections at the same neuroanatomical level (cingulate cortex) were imaged and assessed. A 0.1-mmwide rectangle spanning the cerebral wall was placed over the center of the cingulate cortex (supplemental Fig. S5A, available at www.jneurosci. org as supplemental material). Embryonic zones were identified using fluorescent Nissl staining. The whole thickness of the mPFC was divided into 10 equal bins [bin 1 within the proliferative zone (PZ) and bin 10 within the marginal zone (MZ)] within this rectangle, and TH-positive axon density was assessed within each bin using ImageJ software. Data were normalized to total density per square millimeter and averaged for each embryo, and data from several individual animals were pooled. To better visualize and compare TH innervation of wild-type and mutant $\mathrm{mPFC}$, line drawings based on camera lucida were obtained from three consecutive sections.

To determine the orientation of TH-positive axons within the cortical plate (CP) of the mPFC of wild-type mice and of sema3 $\mathrm{F}^{-1-}, n p n-2^{-1-}$, and $n p n-2^{-1-} ; \mathrm{TH}-\mathrm{Cre}$ mutant mice and their littermate controls, the angle of TH-positive axons relative to the pial surface was determined and quantified in each experimental condition and grouped according to angle $(0-$ $20,20-40,40-60$, and $>60^{\circ}$ ) (see Fig. $9 \mathrm{~K}$ ). To determine the angles of $\mathrm{TH}$-positive axons in the $\mathrm{CP}$, a line connecting the pial membrane with the ventricular zone (VZ) was moved from right to left within the $0.1-$ $\mathrm{mm}$-wide rectangle spanning the cerebral wall of the cingulate cortex 
used for the TH-positive axon density measurements described above. The angle of all $\mathrm{TH}$-positive axons in the $\mathrm{CP}$ within the rectangle was subsequently determined in relation to this line using OpenLab software (Improvision) (supplemental Fig. S5A, available at www.jneurosci.org as supplemental material). As described above, three to five embryos were analyzed per developmental stage and two to four well spaced $(80 \mu \mathrm{m})$ sections at the same neuroanatomical level were imaged and assessed. $0^{\circ}$ corresponds to a trajectory perpendicular to the pial surface, whereas $90^{\circ}$ represents a trajectory parallel to the pia. Furthermore, the first $10 \mathrm{TH}-$ positive axons that crossed the line while it was shifted from right to left within the rectangle were plotted in axon orientation plots (see Fig. 9, shown as insets).

Data (overall axon density, axon density/bin, and percentage of axons/ group) were tested for significance by one-way ANOVA $(\alpha=5 \%)$ and expressed as means \pm SEM.

DiI tracing. For DiI labeling, brains were dissected and fixed by immersion in 4\% PFA in PBS, pH 7.4, containing 4\% sucrose and microinjected with DiI ("paste" preparation; Invitrogen). To ensure small and reproducible DiI injections, small pockets of tracer $(\sim 0.2-0.5 \mu \mathrm{l})$ were microinjected into the rostral-to-caudal extent of the mVTA or mPFC of wild-type or $s \mathrm{ema} \mathrm{F}^{-1-}$ mice (and littermate controls) using a picospritzer (Harvard Apparatus). Brains were stored in fixative for $4-8$ weeks in the dark at room temperature, rinsed in PBS, embedded in 5\% low-melting-point agarose in PBS, and sectioned coronally at 80-100 $\mu \mathrm{m}$ on a Vibratome (Leica VT 1000S). Free-floating sections were then counterstained with either bisbenzamide or DAPI $\left(4^{\prime}, 6^{\prime}\right.$-diamidino-2phenylindole) $(0.1 \mathrm{mg} / \mathrm{ml}$ in PBS; Invitrogen $)$, coverslipped in $90 \%$ glycerol in PBS, and examined by epifluorescence microscopy (Zeiss Axioskop 2) or by confocal laser-scanning microscopy (Zeiss LSM510). Specific designations such as rostral-to-caudal portions of mVTA are explained in Figure 3. The specificity of the DiI injections can be inferred from the observations that injection in the rostral mVTA consistently labeled projections to the $\mathrm{mPFC}$, whereas microinjections in the mPFC led to labeled neurons in the rostral mVTA only. Furthermore, injections in other parts of the mVTA did not mark mesoprefrontal projections but did reproducibly label other mdDA pathways.

Explant cultures. Three-dimensional collagen matrix assays were performed as described previously (Pasterkamp et al., 2003). E12.5 or E14.5 embryos were collected in ice-cold L15 medium and brains were rapidly dissected. Small explants $(<350 \mu \mathrm{m})$ were dissected from (1) the mVTA in a rostral-to-caudal direction, bisected along the midline; (2) the mPFC (prelimbic and cingulate areas); and (3) the somatosensory (S1) cortex (Ctx) (see Fig. 3A) and collected in ice-cold L15 containing 10\% FCS. Combinations of mVTA and mPFC or mVTA and S1 were embedded in close proximity ( $\sim 300 \mu \mathrm{m}$ apart) in a collagen matrix in four-well tissue culture dishes (Nunclon Surface; Nunc). In cue culture experiments, explants were embedded next to aggregates of HEK293 cells transiently transfected with expression constructs for Sema3A, Sema3C, Sema3F, or mock-transfected cells as a control. Explants were cultured in DMEM/ F12 with $10 \%$ FCS, glucose, glutamine, and antibiotics in a humidified incubator at $37^{\circ} \mathrm{C}$ with $5 \% \mathrm{CO}_{2}$ for $56-58 \mathrm{~h}$. Explants were then quickly washed in PBS, fixed in buffered 4\% PFA for $1.5 \mathrm{~h}$, and washed extensively before immunocytochemistry for both $\mathrm{TH}$ and $\beta$ III-tubulin to visualize dopaminergic or all neurites, respectively. In addition, a few explant cultures were immunostained for both $\mathrm{TH}$ and calbindin or $\mathrm{TH}$ and Girk 2 to confirm the identity of the mVTA explants. In blocking experiments, explants were cultured in the presence of either recombinant human IgG-Fc ( $4 \mu \mathrm{g} / \mathrm{ml}$; Calbiochem) as a control, Npn-1-Fc (2 $\mu \mathrm{g} / \mathrm{ml}$; R\&D Systems), or Npn-2-Fc ( $2 \mu \mathrm{g} / \mathrm{ml}$; R\&D Systems). Similar experiments were performed using rabbit Igs, anti-Npn-1, and antiNpn-2 function blocking antibodies (Kolodkin et al., 1997; Giger et al., 1998). The specificity and activity of these blocking tools were confirmed in repulsion assays for individual Sema3s and can also be inferred from the results of previous studies (Kolodkin et al., 1997; Giger et al., 1998; Anderson et al., 2003). For each of the experimental conditions mentioned above (E12.5 and E14.5 cocultures and cue cultures, with and without blocking tools), at least three independent experiments were performed.

For quantification of explant assays, the length of the 20 longest neurites was measured in both the proximal and distal quadrants of the
Table 2. Sense and antisense primer sequences

\begin{tabular}{lll}
\hline Gene & Sense primer & Antisense primer \\
\hline Sema3A & $5^{\prime}$-tcatcatgggcacagtcttg-3' & $5^{\prime}$-catgtgcttccacttgttgc-3' \\
Sema3B & $5^{\prime}$-aagacctttggcacttcag-3' & $5^{\prime}$-tcgccattccttacgttttg-3' \\
Sema3C & $5^{\prime}$-tggaggagctggaagtctt-3' & $5^{\prime}$-ttgaagttgatcttggctatgg-3' \\
Sema3D & $5^{\prime}$-ggaacagcttctgatttccttg-3' & $5^{\prime}$-gccaaaatcaccttttcatc-3' \\
Sema3E & $5^{\prime}$-agtgcttgtgctgactgctg-3' & $5^{\prime}$-ttccactctctggaagttgc-3' \\
Sema3F & $5^{\prime}$-tggaaccgaacacacctgta-3' & $5^{\prime}$-tctgcactgtcagggatgag-3' \\
Sema3G & $5^{\prime}$-cagtgcgctggttcttacaa-3' & $5^{\prime}$-gcaccctgctcttcatcttc-3' \\
$\beta$-Actin & $5^{\prime}$-agaagagctatgagctgcctga-3' & $5^{\prime}$-tacttgcgctcaggaggagcaa-3' \\
\hline
\end{tabular}

culture using OpenLab software (Improvision). The average value of each explant was used to determine the proximal/distal ratio (P/D ratio) per explant. Data were statistically analyzed by one-way ANOVA $(\alpha=$ $5 \%)$ and expressed as means \pm SEM.

Reverse transcriptase-PCR. To study the expression of Sema3s in specific brain areas, reverse transcriptase (RT)-PCR was performed. Targeting primers were designed using Primer3 software (Whitehead Institute for Biomedical Research, Cambridge, MD) (Table 2). In brief, total RNA from E14.5 and E17.5 whole head and MPFC was extracted using TRIzol reagent (Invitrogen) according to the manufacturer's protocol. A total of $25 \mathrm{ng}$ of total RNA was used for cDNA synthesis $\left(50^{\circ} \mathrm{C}\right.$ for $30 \mathrm{~min}$, followed by a $15 \mathrm{~min}$ initial denaturation step at $95^{\circ} \mathrm{C}$ ) and gene amplification reactions using SuperScript One-Step (Invitrogen) in a total volume of $50 \mu \mathrm{l}$. PCR amplification was conducted at the following temperatures and times: $94^{\circ}$ for $1 \mathrm{~min} ; 56^{\circ}$ for $1 \mathrm{~min}, 72^{\circ}$ for $1 \mathrm{~min}$ for either 27 or 33 cycles; $72^{\circ}$ for $10 \mathrm{~min}$. Controls were included to monitor specificity. PCR products were size-separated on a $1.5 \%$ agaroseethidium bromide gel, extracted, and verified by sequencing.

In situ hybridization. Nonradioactive in situ hybridization was performed according to Pasterkamp et al. (2007). Digoxigenin-labeled cRNA probes were transcribed from mouse $n p n-1$ (a 303 bp fragment corresponding to nucleotides $271-574$ of the coding region), mouse $n p n-2$ (a 297 bp fragment corresponding to nucleotides $946-1139$ of the coding region), or rat $T H$ (nucleotides $915-1137$ of the coding region) (Pasterkamp et al., 2007) cDNAs. Probes transcribed from the entire coding region of rat sema $3 A \mathrm{cDNA}$, rat sema $3 \mathrm{C} \mathrm{cDNA}$, and rat sema3F cDNA were alkali-hydrolyzed [a kind gift from Dr. J. Verhaagen (Netherlands Institute for Neuroscience, Amsterdam, The Netherlands) and Dr. A. W. Püschel (Westfalische Wilhelms-Universität Münster, Münster, Germany), respectively]. Sections subjected to the entire in situ hybridization procedure, but with no probe or sense probe added, did not exhibit specific hybridization signals. The specificity of the in situ hybridization procedure was also inferred from the clearly distinct sema 3 and $n p n$ distribution patterns.

\section{Results}

\section{Development of mesoprefrontal mdDA axon projections in the mouse}

mdDA neurons give rise to three major axonal pathways. In this study, we focused on one of these pathways (the mesoprefrontal pathway) to further determine the molecular program that controls mdDA pathway development in vivo. The embryonic origin and ontogeny of mouse mesoprefrontal mdDA projections is mostly unknown. Therefore, we first generated a detailed map of mesoprefrontal mdDA projections during mouse development using immunocytochemistry for $\mathrm{TH}$, the rate-limiting enzyme in dopamine synthesis, and lipophilic dye tracing.

At E11.5, TH-positive neurons lining the mesencephalic flexure (mf) started to extend neurites dorsally (Fig. $1 A, B$, arrowheads). At E13.5, a TH-positive axon tract had formed, the MFB, projecting into the forebrain (Fig. $1 C$ ). As early as E14.5, THpositive axons reached a region ventral to the medial and lateral ganglionic eminences (MGE and LGE, respectively) (Fig. 1D). Whereas most axons extended dorsally at this choice point to- 

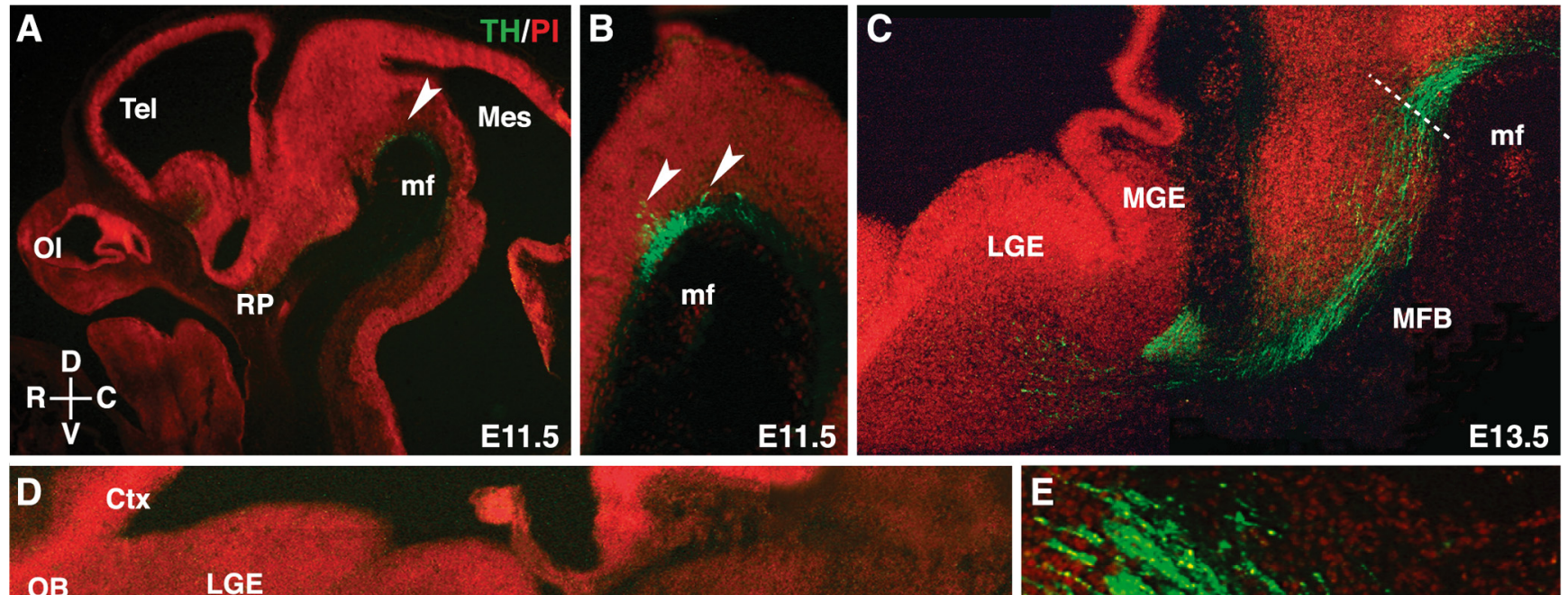

OB LGE

MGE

DT

VT

E14.5
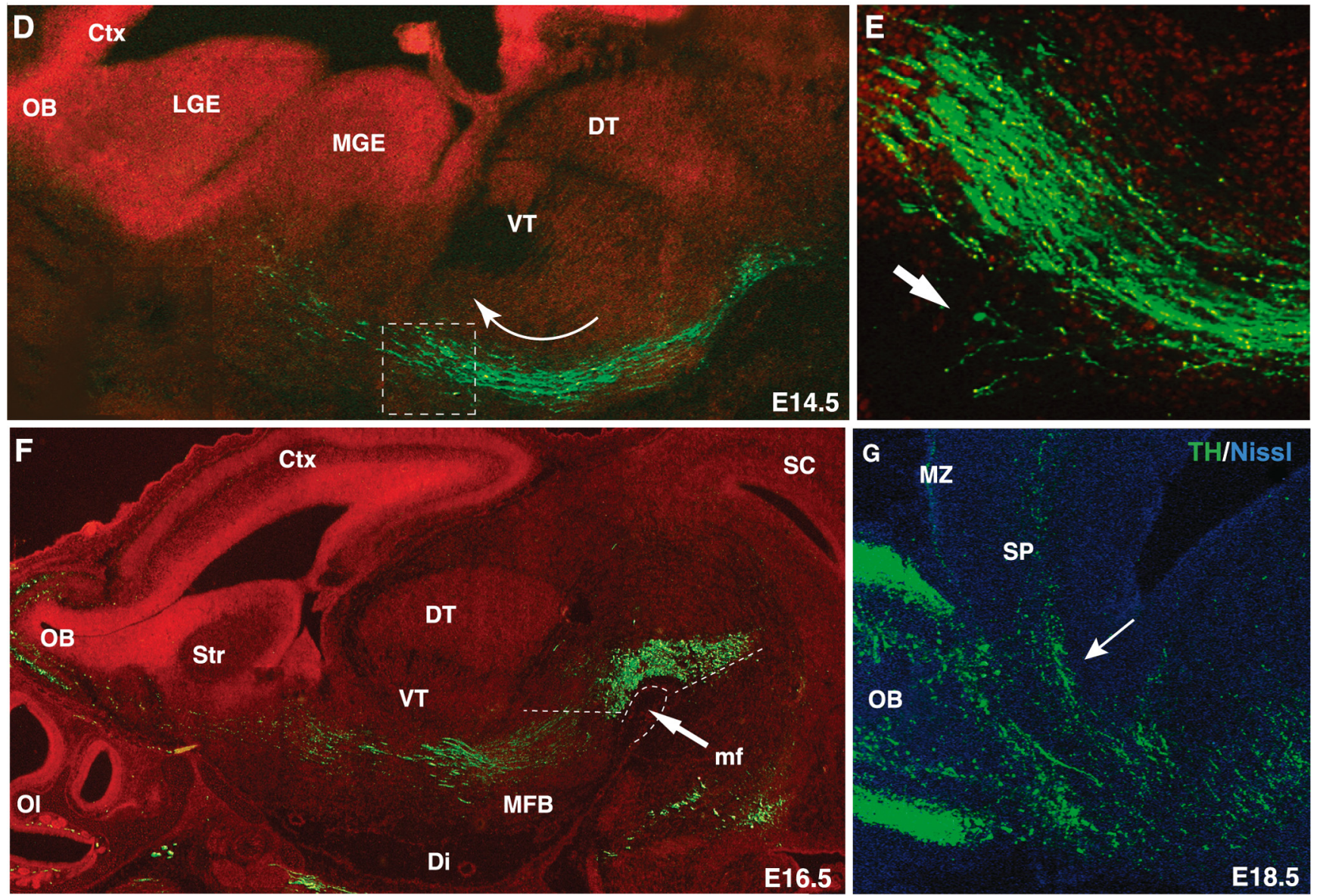

SP

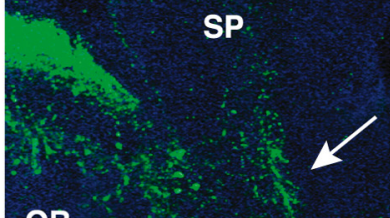

OB

E18.5

Figure 1. Ontogeny of mdDA axon projections in the mouse forebrain. Sagittal sections are immunostained for TH (green) to visualize dopaminergic axons and counterstained with either PI (red) or fluorescent Nissl (blue). $A, B$, At E11.5, TH-positive neurons emerge within the ventral midbrain lining the mesencephalic flexure (mf) and project neurites dorsally (arrowheads). $C$, By E13.5, the MFB has formed and takes a ventrolateral course in the diencephalon. The dotted line indicates rostral (diencephalic) boundary of mdDA system. $\boldsymbol{D}$, At E14.5, the MFB curves dorsally (bent arrow) and reaches the striatum. E represents a higher magnification of the boxed region in $\boldsymbol{D}$. $\boldsymbol{E}$, A subset of TH-positive axons diverges from the main MFB trajectory and extends rostrally. $\boldsymbol{F}$, At $E 16.5$, axons in the MFB are fasciculated into thick axon bundles. G, TH-positive axons curve dorsally just caudal of the OB to enter the cortical SP (arrow). C, Caudal; Ctx, cortex; D, dorsal; Di, diencephalon; DT, dorsal thalamus; LGE, lateral ganglionic eminence; Mes, mesencephalon; MGE, medial ganglionic eminence; MZ, marginal zone; 0l, olfactory epithelium; R, rostral; RP, Rathke's pouch; SC, superior colliculus; Str, striatum; Tel, telencephalon; V, ventral; VT, ventral thalamus.

ward the developing striatum, a small subset of axons continued to project toward more rostral regions such as the mPFC (Fig. $1 E$, arrow). From E16.5 onward, the MFB became more robust and individual $\mathrm{TH}$-positive axons within the MFB were fasciculated into thick axon bundles (Fig. $1 F$ ). To reach the mPFC, THpositive axons followed two distinct trajectories. A large subset of $\mathrm{TH}$-labeled axons redirected dorsally just caudal of the olfactory bulb (OB) to extend toward the MPFC (Fig. 1G, arrow). Another, smaller, subset of $\mathrm{TH}$-labeled axons traversed the striatum, crossed the developing external capsule (EC), and then innervated the overlaying $\mathrm{mPFC}$ (see Fig. $8 R$ ) (data not shown).

Around E15, the first TH-positive axons were observed within the subplate (SP) and the MZ (arrowheads) of the MPFC (Fig. $2 A, B)$. Although the innervation of the SP and MZ had increased at E16.5, the CP remained devoid of TH-positive axons (Fig. 2C). Previous reports demonstrated a waiting period for mesoprefrontal mdDA axons in the SP of rat and human PFC (Kalsbeek et al., 1988; Verney, 1999). Consistent with these results, the mouse 
A
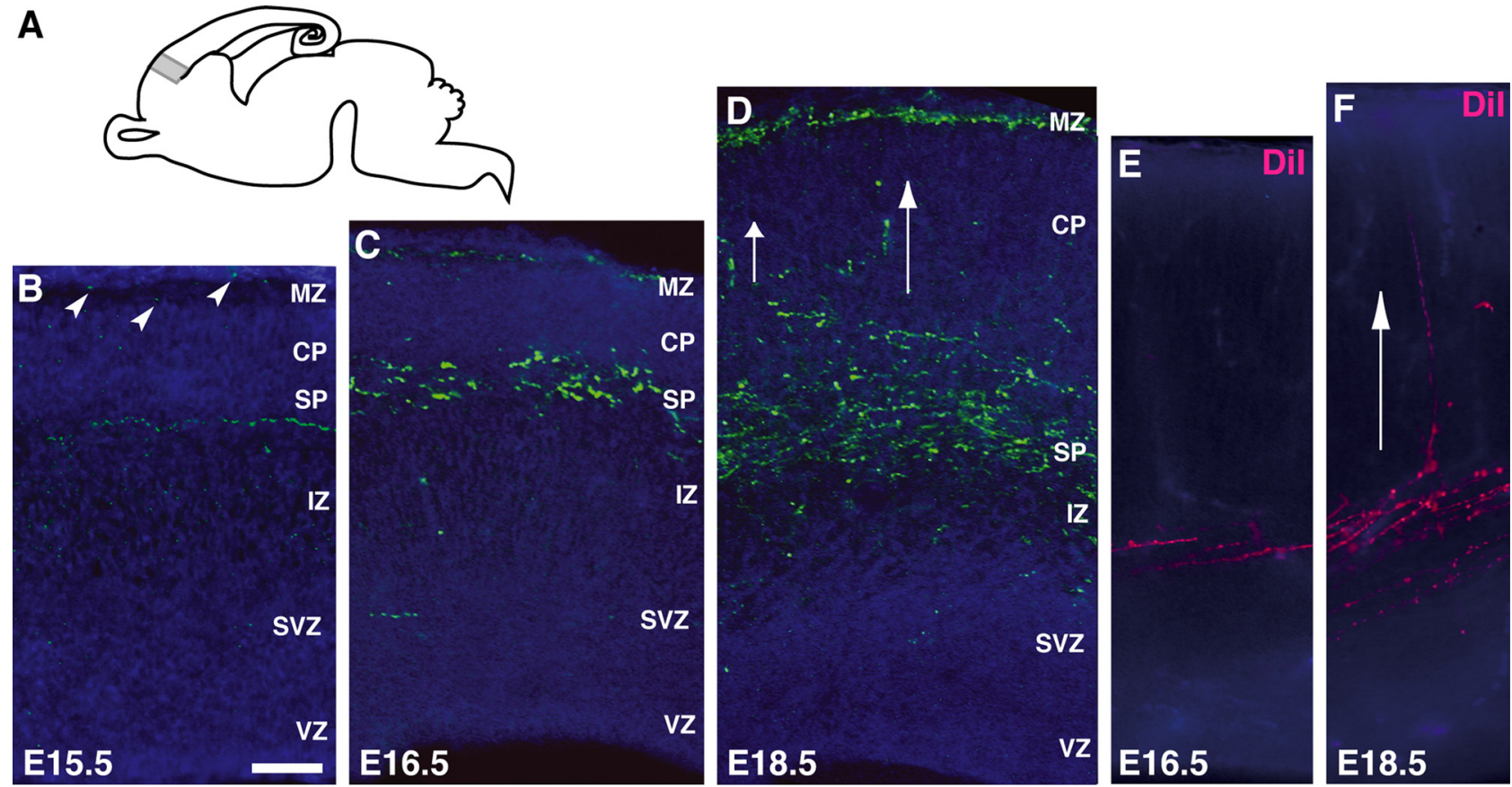

Figure 2. mdDA neurons in the rostral mVTA project axons to the mPFC during development. $\boldsymbol{A}$, Schematic showing in gray the derivation of the $\mathrm{mPFC}$ sections shown in $\boldsymbol{B}-\boldsymbol{F}$. $\boldsymbol{B}-\boldsymbol{D}$, Sagittal sections are immunostained for TH (green) to visualize mdDA axons and counterstained with fluorescent Nissl (blue). $\boldsymbol{B}$, TH-positive axons start to enter the embryonic cortex around E15.5 and travel tangentially within the SP. In addition, some TH-positive puncta can be observed within the MZ (arrowheads). C, At E16.5, fibers appear within the SP. However, the CP is still mostly devoid of TH-positive axons. D, By E18.5, axons extend from the SP to the (P following a radial trajectory (arrows). $\boldsymbol{E}, \boldsymbol{F}$, To identify the origin of mesoprefrontal mdDA projections during development, Dil tracer was microinjected in the medial aspect of the VTA at different developmental time points (for an overview of all injection sites, see supplemental Fig. S1, available at www.jneurosci.org as supplemental material). Coronal sections are counterstained with Hoechst (blue) and show Dil in red. $\boldsymbol{E}, \boldsymbol{F}$, After injections in the rostral mVTA, but not in more lateral, middle, or caudal aspects of the mVTA, Dil-labeled axons were found in the mPFC. At E16.5, Dil-positive axons derived from rostral mVTA enter the mPFC through the SP $(\boldsymbol{E})$ and start to innervate the $C P$ around E18.5 $(\boldsymbol{F}$, arrow). IZ, Intermediate zone; VZ, ventricular zone. Scale bar: $\boldsymbol{B}-\boldsymbol{F}, 100 \mu \mathrm{m}$.

$\mathrm{CP}$ became permissive for $\mathrm{TH}$-positive axons after a waiting period of $\sim 2 \mathrm{~d}$ within the SP (E15-E17.5). At E18.5, TH-positive axons invaded the CP from the underlying SP following a characteristic radial trajectory (Fig. $2 D$, arrows). In postnatal and adult $\mathrm{mPFC}$, TH-positive axons were present in all layers but most abundantly in layers I, V, and VI (data not shown).

\section{Mesoprefrontal mdDA projections originate in the rostral VTA during early development}

$\mathrm{TH}$ labels both dopaminergic and noradrenergic nuclei and fails to discriminate between different mdDA pathways. In the adult mouse, mesoprefrontal mdDA projections originate in the medial VTA (Fallon, 1981; Swanson, 1982; Margolis et al., 2006; Lammel et al., 2008). To more specifically determine the embryonic origin and ontogeny of mesoprefrontal mdDA projections, microinjections of DiI were placed along the rostrocaudal axis of the medial VTA (designated mVTA) (Fig. $3 A$ ) in fixed embryonic brains (E15.5-E18.5). After injections in the rostral mVTA, but not in more lateral, middle, or caudal aspects of the developing mVTA, DiI-labeled axons were found in the mPFC (Fig. 2E, F; supplemental Fig. S1 $A-E$, available at www.jneurosci.org as supplemental material). Interestingly, in the adult mouse mdDA mesoprefrontal neurons are located in a middle subdivision of the mVTA (Lammel et al., 2008) (data not shown). This suggests that these neurons eventually become confined to a more caudal aspect of the VTA as a result of ongoing developmental processes (e.g., cell migration) (Smidt and Burbach, 2007). Innervation of the SP by DiI-labeled axons was clearly observed around E16 ( $n=$ 5) (Fig. $2 E$ ). In line with the waiting period noted for TH-positive axons (Fig. $2 B-D$ ), DiI-labeled rostral mVTA axons only began to innervate the CP of the mPFC around E18.5 $(n=6)$ (Fig. $2 F$, arrow). DiI-labeled axons innervating the $\mathrm{mPFC}$ expressed $\mathrm{TH}$, confirming their dopaminergic nature (data not shown). Interestingly, no DiI-positive axons were observed in the $\mathrm{MZ}$ of the mPFC after mVTA injections, suggesting that TH-positive axons in the MZ (Fig. $2 B-D$ ) do not derive from the mVTA (Fig. $2 E, F$ ). The trajectory of DiI-labeled rostral mVTA axons in the forebrain was consistent with observations for TH-positive axons (Fig. $1 D-G$; supplemental Fig. $S 1 F-H$, available at www.jneurosci.org as supplemental material). To further study neuronal connections between the VTA and mPFC, DiI injections were placed in the developing mPFC (supplemental Fig. S1 $J-L$, available at www.jneurosci.org as supplemental material). DiI injections in E16.5 and P0 mPFC traced back to small, rounded cells in the rostral and middle parts of the mVTA $(n=7)$ (supplemental Fig. $\mathrm{S} 1 M, N$, available at www.jneurosci.org as supplemental material). The appearance and size of these cells was reminiscent of mdDA VTA neurons (Margolis et al., 2006). Collectively, our data show that, during embryonic development, mdDA neurons in the rostral part of the mVTA project their axons along a highly stereotypic trajectory to the mPFC.

The mPFC releases diffusible molecules that exert directional effects on mVTA axon outgrowth

To establish precise connections, axons rely on heterogeneously distributed molecular cues in their environment (TessierLavigne and Goodman, 1996; Dickson, 2002; Huber et al., 2003; Petros et al., 2008). The molecules that help to establish mesoprefrontal mdDA connections have yet to be determined. As a first step toward identification of these molecular cues, coculture as- 
A
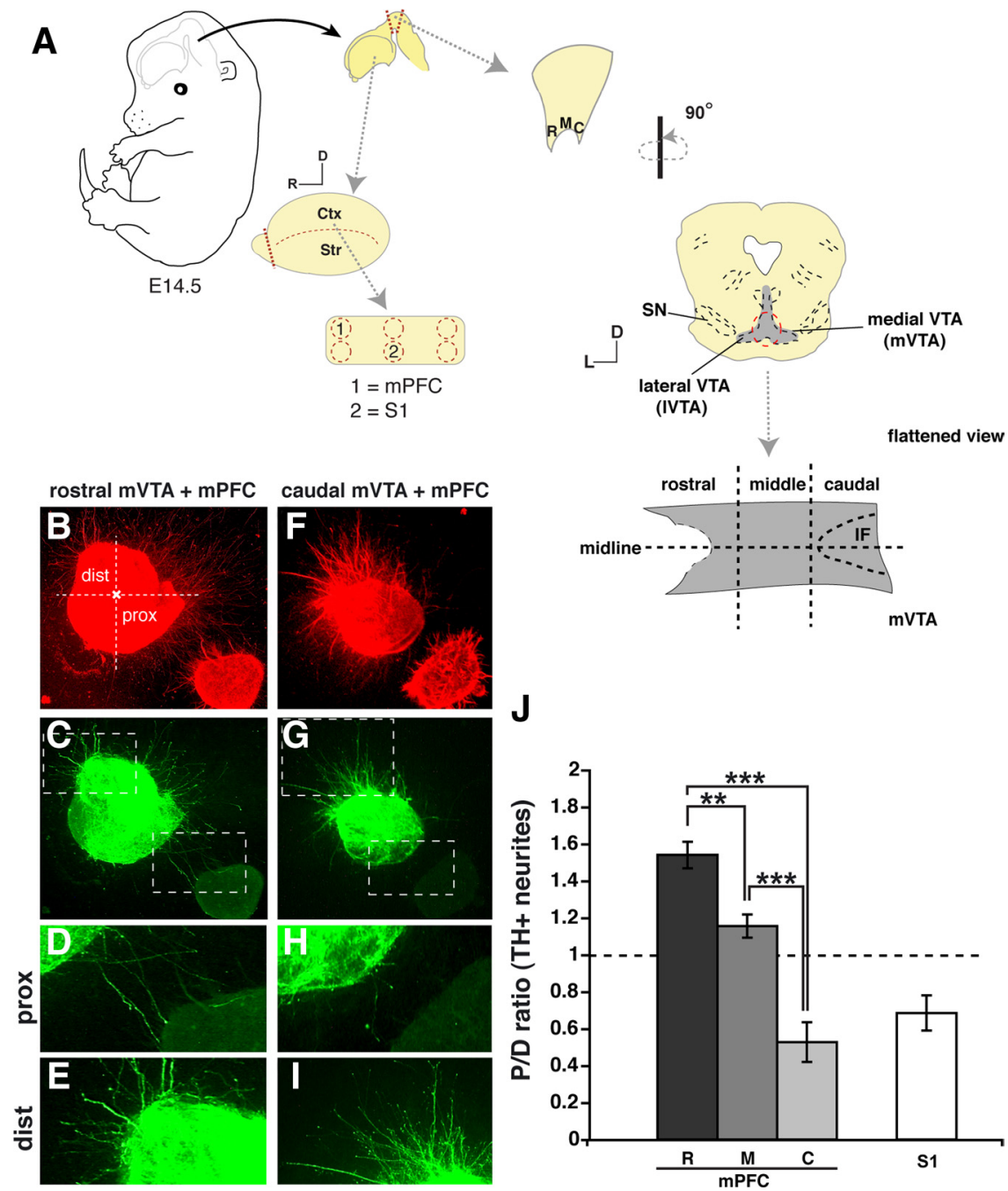

$\mathbf{J}$

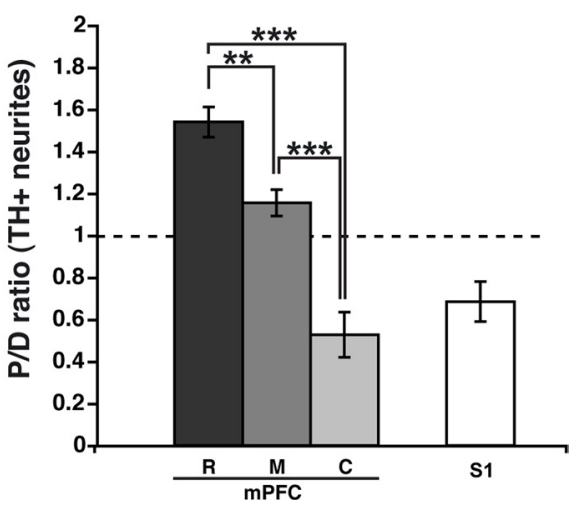

Figure 3. The $\mathrm{mPFC}$ releases diffusible molecules that exert neuronal subset-specific directional effects on mVTA axon outgrowth. $\boldsymbol{A}$, Schematic representation of the coculture system used to identify axon guidance activities between cortex and mVTA. Explants of E14.5 embryos were taken from the ventral midbrain along the rostrocaudal axis (mVTA), the mPFC, and somatosensory (S1) cortex. Explants were analyzed after 56 - 58 h in culture using immunohistochemistry for TH and $\beta$ III-tubulin to visualize dopaminergic (green) and all (red) neurites, respectively. Rostral (R), middle (M), or caudal (C) parts of mVTA explants were cocultured with mPFC ( $n=$ 8,10 , and 9 , respectively) and $S 1(n=8) . \boldsymbol{B}-\boldsymbol{E}, \boldsymbol{J}$, Cocultures of rostral mVTA and mPFC show attraction of TH-positive axons. $\boldsymbol{F}-\boldsymbol{J}$, In contrast, TH-positive axons extending from the caudal mVTA are strongly repelled. The boxed areas in $\boldsymbol{C}$ and $\mathbf{G}$ are shown in $\boldsymbol{D}, \boldsymbol{E}, \boldsymbol{H}$, and $\boldsymbol{I}$. $\boldsymbol{J}$, Quantification of the length of TH-positive neurites in the proximal and distal quadrants of the coculture assays as shown in $\boldsymbol{B}-\mathbf{I}$. Graph shows average $P / D$ ratio $\pm S E M$. TH-positive axon outgrowth from the $m V T A$ in the presence of $m P F C$ is shown separately for the $R$, $M$, and C parts of the mVTA. For S1 P/D ratios for $R, M$, and C explants are pooled. For each experimental condition, at least three independent experiments were performed. ${ }^{* *} p<0.01$; ${ }^{* * *} p<0.001$, one-way ANOVA. Ctx, Cortex; dist, distal; D, dorsal; IF, interfascicular nucleus; $\mathrm{L}$, lateral; prox, proximal; SN, substantia nigra; Str, striatum. Scale bar, $250 \mu \mathrm{m}$.

says were developed combining explants of the rostral, middle, or caudal part of the embryonic mVTA and the mPFC (Fig. 3A). To unequivocally demonstrate that TH-positive axons in these cultures derive from the VTA and not from the adjacent SN, a series of cultures were double-stained for TH and calbindin, a marker for VTA mdDA neurons, or for TH and Girk2, a marker for SN mdDA neurons (supplemental Fig. S2, available at www. jneurosci.org as supplemental material) (Lin et al., 2005; Thompson et al., 2005). Indeed, TH-positive mVTA explants coexpressed the VTA marker calbindin but not Girk2 (supplemental Fig. S2, available at www.jneurosci.org as supplemental material). Axons extending from explants dissected from the rostral and to a lesser extent middle part of the mVTA were attracted by the $\mathrm{mPFC}$ (Fig. $3 B-E, J)(\mathrm{P} / \mathrm{D}$ ratio, 1.53 and 1.17 , respectively; $n=8$ and 10 ; both $p<0.001$ compared with caudal mVTA). In contrast, axons from caudal mVTA explants were robustly repelled (Fig. $3 F-I, J$ ) (P/D ratio, $0.53 ; n=9 ; p<0.001)$. A strong repulsive effect was also observed when mVTA explants were cocultured with the somatosensory cortex (S1), an area only sparsely innervated by mVTA axons in vivo (Fig. $3 J)(\mathrm{P} / \mathrm{D}$ ratio, 0.68 ; $n=8$ ). These data show that the mPFC releases diffusible cues that differentially affect the directional outgrowth of axons derived from distinct parts of the mVTA. mdDA axons from rostral mVTA that normally target the mPFC, as shown by our immunohistochemical and DiI data, are attracted by the mPFC.

Neuropilin- 2 mediates the directional
growth of rostral mVTA axons induced by the MPFC

The data from our coculture experiments suggest that the $\mathrm{mPFC}$ releases molecular cues that specifically attract mdDA axons from rostral mVTA neurons. In an attempt to identify these molecular signals, we focused on class 3 semaphorins (Sema3s). Sema3s display highly dynamic patterns of expression in the developing cerebral cortex (Giger et al., 1996; Bagnard et al., 1998; Skaliora et al., 1998; Pascual et al., 2005) and exert chemotropic effects on mdDA axons in vitro (Hernández-Montiel et al., 2008; Yamauchi et al., 2009). Furthermore, elegant recent work by Yamauchi et al. (2009) suggests that expression of Sema3F at the midbrain-hindbrain boundary (MHB) determines the polarity of early (E11E12) embryonic mdDA projections within the midbrain. If Sema3s are involved in mesoprefrontal mdDA axon growth and guidance, then Sema3 receptors should be expressed in MVTA neurons. To test this hypothesis, we studied the expression of the Sema3 ligandbinding receptors, Npn-1 and Npn-2, in the mVTA at the time of axon pathfinding using in situ hybridization (Fig. $4 A-C^{\prime}$ ). At E14.5, $n p n-1$ weakly labeled the mdDA region (Fig. $4 B, B^{\prime}$ ), whereas $n p n-2$ was highly expressed in the rostral mVTA (Fig. $4 C, C^{\prime}$ ). To examine whether rostral mVTA neurons and axons express Npn proteins, we performed double immunohistochemistry for TH and Npn-1 or Npn-2. At the protein level, Npn-1 displayed a general and diffuse pattern of expression in the mVTA (data not shown), whereas Npn-2 labeled a subset of TH-positive neurons in the rostral and middle portion of the mVTA (Fig. $4 D, D^{\prime}$, arrowheads). Furthermore, Npn-2, but not Npn-1, was expressed on THpositive axons in the MFB as well as in the SP of the mPFC (Fig. $4 E-H$, arrowheads). Thus, mdDA neurons in the rostral mVTA that give rise to mesoprefrontal axon projections express Npn-2.

Given its expression in the rostral mVTA, Npn-2 is a candidate receptor for mediating the effects of the MPFC on rostral mVTA axons. To test this possibility, we used soluble Npn-2 and 
A
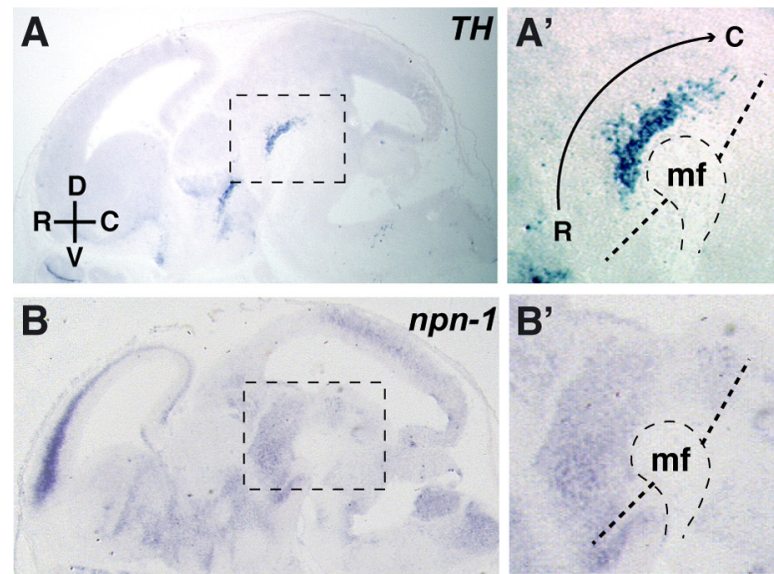

C
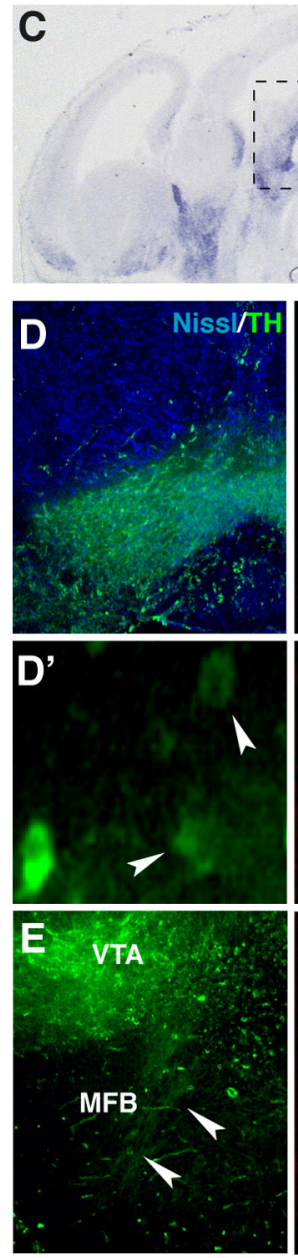

npn-2

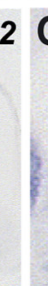

\section{C'}
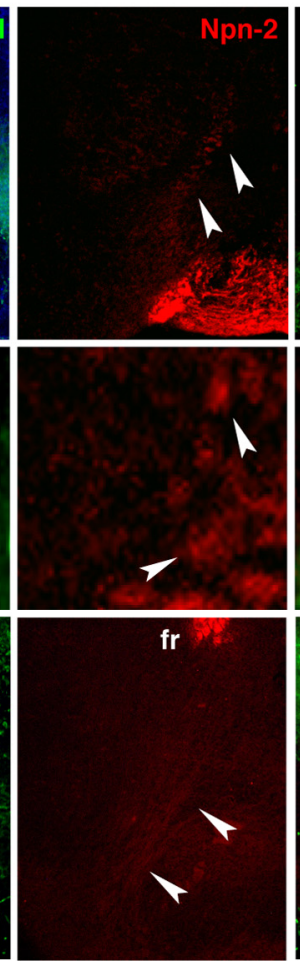

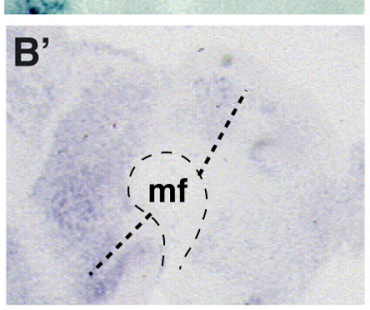

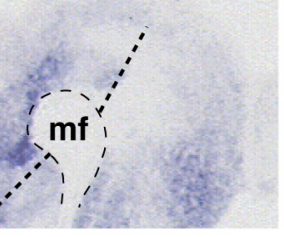
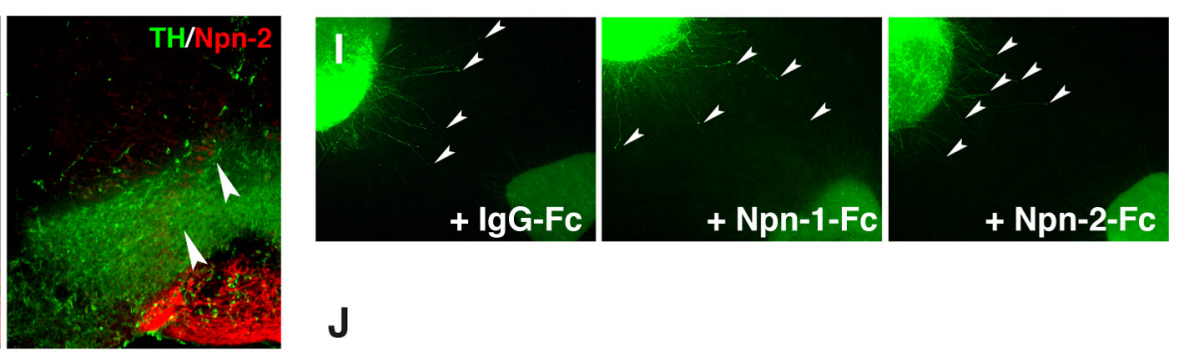

J
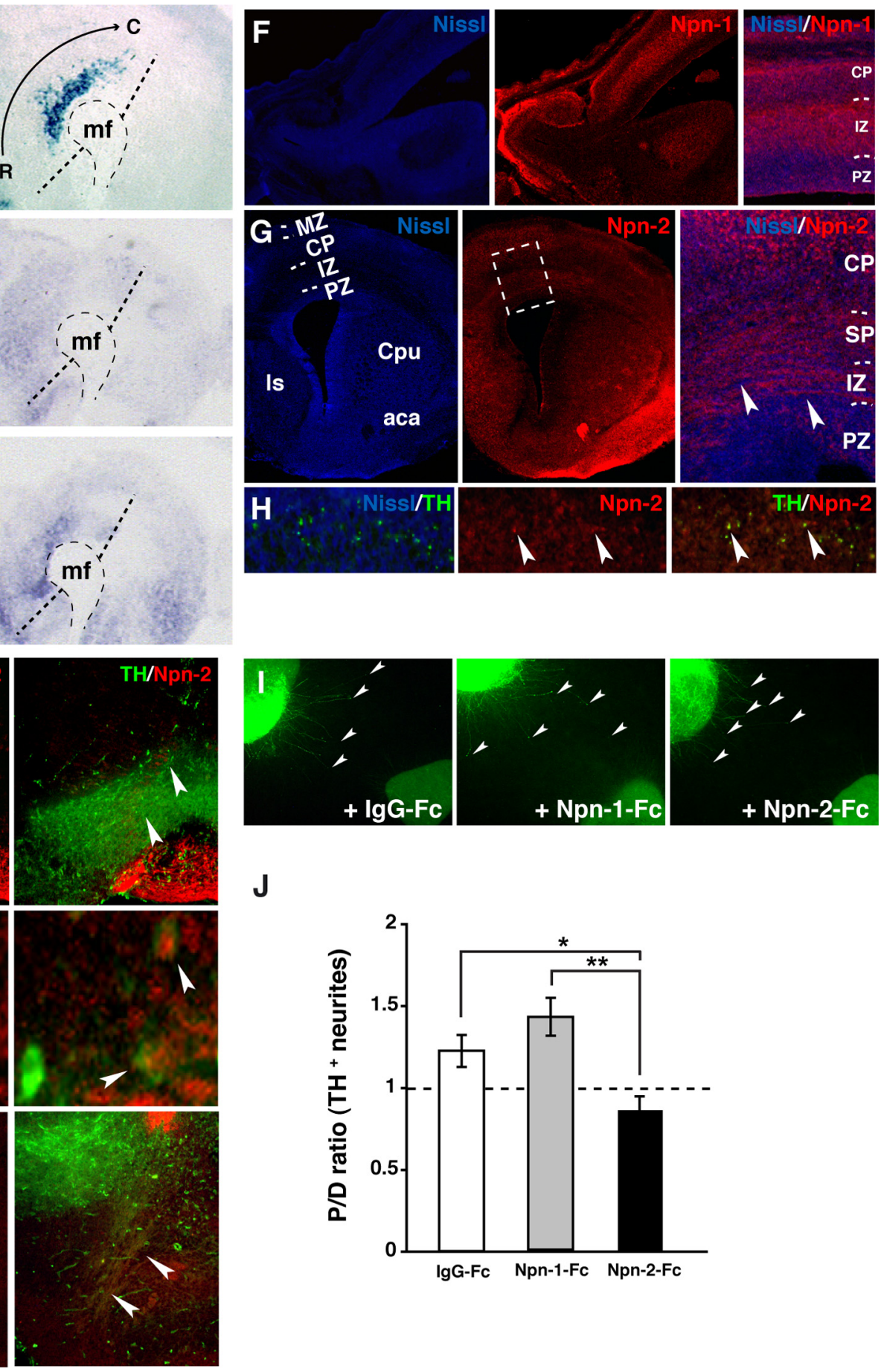

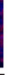

$+\lg G-F c$

+ Npn-1-F

+ Npn-2-Fc

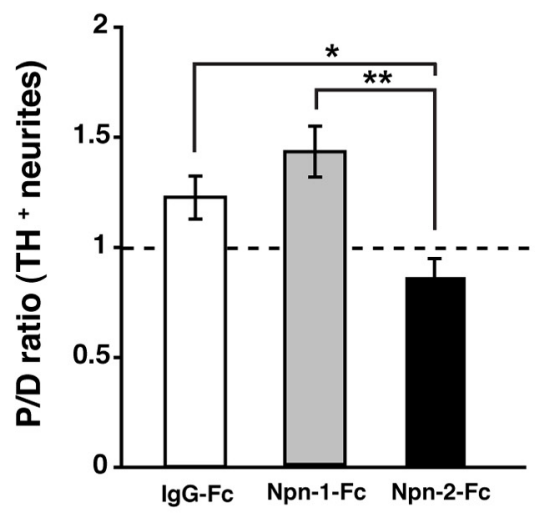

Figure 4. Neuropilin-2 mediates the attractive effect of mPFC on mdDA rostral mVTA axons. $\boldsymbol{A}-\boldsymbol{C}^{\prime}$, In situ hybridization shows expression of $n p n-1(\boldsymbol{B})$ and $n p n-2(\boldsymbol{C})$ within the $T H$-positive $(\boldsymbol{A})$ mdDA system $\left(\boldsymbol{A}^{\prime}-\boldsymbol{C}^{\prime}\right)$. The boxes in $\boldsymbol{A}-\boldsymbol{C}$ are enlarged in $\boldsymbol{A}^{\prime}-\boldsymbol{C}^{\prime}$. Note the expression of $n p n-2\left(\boldsymbol{C}^{\prime}\right)$ in specific neuronal subsets. $\boldsymbol{D}, \boldsymbol{E}$, Immunostaining of coronal sections for TH (green) and Npn-2 (red) counterstained with fluorescent Nissl (blue) shows Npn-2-positive cells ( $\boldsymbol{D}$, arrowheads) within the TH-positive rostral mVTA. $\boldsymbol{D}^{\prime}$, Enlargement of the area indicated by top arrowhead in D shows colocalization of a subset of TH-positive cells with Npn-2 (arrowheads). $\boldsymbol{E}$, Npn-2 (red) is present in the TH-positive MFB. $\boldsymbol{F}$, Npn-1 (red) is present in cells of the IZ and CP. G, Axons within the SP of the mPFC are Npn-2-positive (boxed area enlarged on the right, arrowheads) and colocalize with TH (H, arrowheads). $\boldsymbol{I}$, Representative examples of cocultures of rostral mVTA and mPFC explants in the presence of lgG-Fc, Npn-1-Fc, or Npn-2-Fc immunostained for TH (green). The arrowheads indicate the growth cones (leading edge) of some of the longest TH-positive neurites. $J$, Quantification of the length of TH-positive neurites in the proximal and distal quadrants of rostral mVTA/mPFC cocultures in the presence of $\operatorname{lgG}-\mathrm{Fc}(n=13), \mathrm{Npn}-1-\mathrm{Fc}(n=12)$, or Npn-2-Fc $(n=$ 9). Graph shows average P/D ratio \pm SEM. Three independent blocking experiments were performed. ${ }^{*} p<0.05$; ${ }^{* *} p<0.01$, one-way ANOVA (compared with control lgG-Fc). Note the ability of Npn-2-Fc to block the positive effect of mPFC on rostral mVTA axons. aca, Anterior commissure; CP, cortical plate; CPu, caudate-putamen; D, dorsal; fr, fasciculus retroflexus; IZ, intermediate zone; $\mathrm{Is}$, lateral septum; $\mathrm{mf}$, mesencephalic flexure; $\mathrm{MZ}$, marginal zone; $\mathrm{PZ}$, proliferative zone; $\mathrm{V}$, ventral.

Npn-1 (as a control) ectodomains fused to the human Fc domain and antibodies to functionally block Npns (Kolodkin et al., 1997; Giger et al., 1998; Anderson et al., 2003; Falk et al., 2005; Osborne et al., 2005; Hernández-Montiel et al., 2008) in cocultures of
mPFC and rostral mVTA (Fig. 4I,J). When mPFC and rostral mVTA were cocultured in the presence of either IgG-Fc, Npn-1Fc, or Npn-2-Fc, Npn-2-Fc but not IgG-Fc or Npn-1-Fc dramatically blocked the positive effect of the mPFC on rostral mVTA 


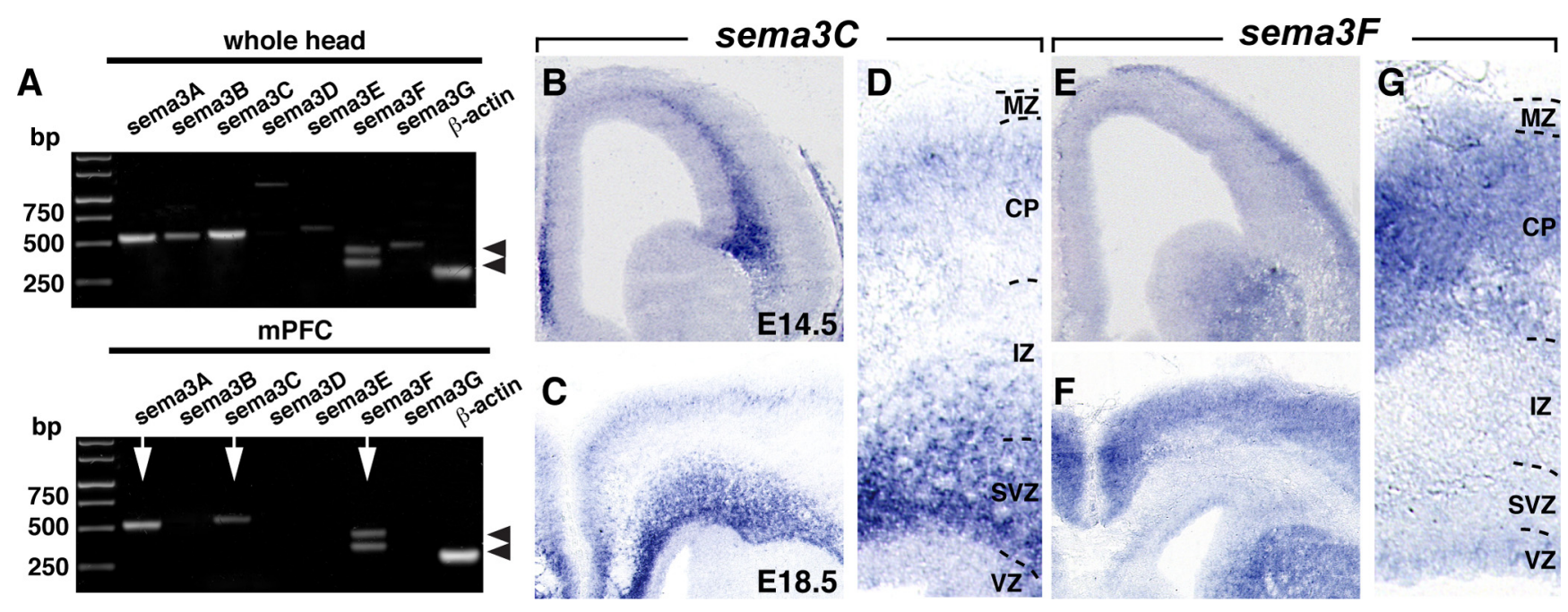

Figure 5. Expression of Sema3s in the mPFC. $A, R T-P C R$ showing the expression of sema $3 A, 3 B, 3 C, 3 D, 3 E, 3 F$, and $3 G$ in mouse head and $\mathrm{mPFC}$ at $E 14.5$. Only sema $3 A, 3 C$, and $3 F$ can be found in the mPFC (arrows). Splice forms of sema3F are indicated by arrowheads (Kusy et al., 2003). Actin serves as a control. $\boldsymbol{B}-\mathbf{G}$, In situ hybridization on coronal sections shows sema3 and sema3F expression in the E14.5 $(\boldsymbol{B}, \boldsymbol{E})$ and E18.5 $(\boldsymbol{C}-\boldsymbol{F}) \mathrm{mPFC}$. Sema3C is expressed within the SVZ and IZ and weakly within the upper layers of the $C P$, whereas sema3F is almost exclusively expressed in the $C P$.

axons (Fig. $4 I, J)$ (IgG-Fc, $n=13$; Npn-1-Fc, $n=12$; Npn-2-Fc, $n=9 ; p<0.05)$. Similar results were obtained in cocultures in which Npn function was blocked using function-blocking antiNpn-1 and Npn-2 antibodies (Kolodkin et al., 1997; Giger et al., 1998) (data not shown). Interestingly, the chemorepulsive effect of S1 on mVTA axons could not be blocked using Npn-2-Fc [IgG-Fc, P/D ratio, $0.70(n=10) ; \mathrm{Npn}-2-\mathrm{Fc}, \mathrm{P} / \mathrm{D}$ ratio, $0.67(n=$ $10), p=0.77$ ], whereas Npn-2-Fc converted the axon-repulsive effect observed in caudal mVTA-mPFC cocultures into attraction $[\mathrm{IgG}-\mathrm{Fc}, \mathrm{P} / \mathrm{D}$ ratio, $0.71(n=7) ; \mathrm{Npn}-2-\mathrm{Fc}, \mathrm{P} / \mathrm{D}$ ratio, 1.36 $(n=9), p<0.05]$. Together, these results indicate that Npn-2 mediates the attractive effect of the mPFC on rostral mVTA axons.

\section{Sema3s are expressed in the developing mPFC}

The presence of Npn-2 on mdDA rostral mVTA axon projections, together with the ability of Npn-2 blocking tools to neutralize the positive effect of mPFC on rostral mVTA axons suggests that Npn-2 ligands such as Sema3s mediate the directed axonal outgrowth from the rostral mVTA in the presence of $\mathrm{mPFC}$. To test this idea, we first determined the expression of Sema3s in the mPFC during embryonic development using RTPCR. Of all seven Sema3s that have been identified, only Sema3A, $3 \mathrm{C}$, and $3 \mathrm{~F}$ were detected in the embryonic mPFC at E14.5 and E18.5 (Fig. 5A) (data not shown). Of these three Sema3s, only Sema3C and Sema3F have been shown to signal through Npn-2 (Chen et al., 1997; Kolodkin et al., 1997; Giger et al., 1998). Therefore, we studied the expression of sema3C and sema3F in more detail using in situ hybridization. In situ hybridization revealed expression of sema3C and sema3F within the embryonic mPFC (Fig. 5B-G). At E14.5, sema3C was expressed at the subventricular zone (SVZ)/intermediate zone (IZ) boundary. In contrast, sema $3 F$ displayed high expression in the upper $\mathrm{CP}$ (Fig. $5 B, E$ ). At E18.5, sema3C was present in the SVZ and IZ and was weakly expressed in the upper CP of the mPFC (Fig. 5C,D). Sema3F was expressed within the CP (Fig. 5F, G). Together, these results establish that sema3C and sema3F are expressed in the $\mathrm{MPFC}$ at the time of mesoprefrontal axon innervation.

\section{Sema3F is a bifunctional axon guidance cue for rostral} mVTA axons

To determine whether Sema3C and Sema3F influence mVTA axon outgrowth, E14.5 mVTA explants were cultured next to 293HEK cell aggregates secreting either control, Sema3C, or Sema3F proteins (Fig. 6A). When explants from rostral mVTA were combined with control or Sema3C-expressing cells, no chemotropic axon responses were detected (Fig. $6 A, B)(\mathrm{P} / \mathrm{D}$ ratios, $1.09, n=9$, and 1.01, $n=10$, respectively). In striking contrast, $\mathrm{TH}$-positive axons from rostral mVTA were significantly longer in the proximal compared with the distal quadrant in the presence of Sema3F, suggesting an axon-attractive effect (Fig. $6 A, B)(\mathrm{P} / \mathrm{D}$ ratio, $1.33 ; n=11 ; p<0.05)$. This effect was neutralized by Npn-2 function-blocking tools (Fig. 6B) (P/D ratio, $1.07 ; n=9 ; p<0.05$ ) (data not shown). To provide additional evidence for a role for Sema3F in the formation of mesoprefrontal projections, the ability of alkaline phosphatasetagged Sema3F ligand (Sema3F-AP) to bind the distinct portions of the mesoprefrontal system was examined using section binding (supplemental Fig. S3, available at www. jneurosci.org as supplemental material). In line with the expression and tissue culture data (Figs. 4-6), prominent Sema3F-AP binding was observed in the MVTA region and MFB (1) as well as in the mPFC (2) (supplemental Fig. S3, available at www. jneurosci.org as supplemental material). Together, these data suggest that Sema3F, through binding with Npn-2, acts as an axon attractant for mesoprefrontal mdDA axons.

Remarkably, when explants from E14.5 caudal mVTA were confronted with Sema3F, TH-positive axons were strongly repelled (Fig. $6 B$ ) (P/D ratio, $0.56 ; n=12 ; p<0.001)$. Previous studies show that, similar to caudal mVTA axons, early embryonic rat mdDA axons are repelled by Sema3F (E13.5, analogous to $\sim$ E12 in mice) (Hernández-Montiel et al., 2008; Yamauchi et al., 2009). A possible model to explain why early rat mdDA axons are repelled by Sema3F whereas E14.5 rostral mVTA axons are attracted, is that rostral $\mathrm{mVTA}$ axons change their responsiveness to Sema3F as they develop. To test this intriguing hypothesis, we cultured early embryonic (E12.5) mouse rostral mVTA explants next to cell aggregates secreting control or Sema3F proteins. In- 
terestingly, Sema3F strongly repelled THpositive axons emerging from E12.5 rostral mVTA explants (Fig. $6 C$ ) (P/D ratio, $0.52 ; n=11 ; p<0.001)$. Collectively, these experiments identify Sema3F as a bifunctional guidance cue and show that rostral mVTA axons change their responsiveness to Sema3F from repulsion to attraction as development progresses.

\section{Abnormal mdDA axon channeling and fasciculation in sema3F and $n p n-2$} mutant mice

The ability of Sema3F to repel early mesoprefrontal mdDA projections in vitro (Fig. $6 C$ ) together with the expression of sema3F in several brain regions surrounding the (presumptive) trajectory of the mesoprefrontal mdDA pathway during early development (Fig. 5E; supplemental Fig. S6, available at www.jneurosci.org as supplemental material) (data not shown) (Chen et al., 1997; Giger et al., 1998; Funato et al., 2000; Marín et al., 2001; Tamamaki et al., 2003; Hernández-Montiel et al., 2008; Ito et al., 2008; Yamauchi et al., 2009) suggests that Sema3F might function to guide mesoprefrontal and other mdDA axons en route to their forebrain targets. To test this hypothesis, we studied the fate of mdDA axon projections in mice harboring a null mutation in sema3F (Sahay et al., 2003). Sagittal sections of E13.5 sema $3 F^{-1-}$ mice and wild-type littermate controls were immunostained for $\mathrm{TH}$ to visualize mdDA axon projections. In E13.5 wild-type embryos $(n=3)$, THpositive axons were organized into two compact MFBs that projected rostrally (Fig. $7 A, B)$. In sema3 $F^{-1-}$ mice $(n=2)$, however, the MFB was defasciculated and occupied a wider area along the dorsoventral axis (Fig. 7C). These defects were even more apparent at E18.5. At this stage, THpositive axons in the MFB of wild-type embryos $(n=5)$ were organized in multiple tightly fasciculated axon bundles (Fig. $8 B, F, N$ ). In dramatic contrast, axon bundles in the MFB of sema3 $F^{-1-}$ mice $(n=5)$ were severely defasciculated and the MFB had spread out over a larger area, thereby aberrantly projecting into surrounding regions normally expressing sema3F (Fig. 8C, G,O). Despite these abnormalities, however, the overall caudorostral orientation of the MFB was preserved (Fig. $8 A, C, O$ ). To further assess these defects, DiI tracer was microinjected into the mVTA of E18.5 sema3 $\mathrm{F}^{-1-} \mathrm{em}$ bryos $(n=5)$ and littermate controls $(n=4)$ after which axons in the MFB were visualized and quantified using confocal microscopy. In line with the results described above for $\mathrm{TH}$ immunohistochemistry, DiI-labeled axon bundles within the MFB were
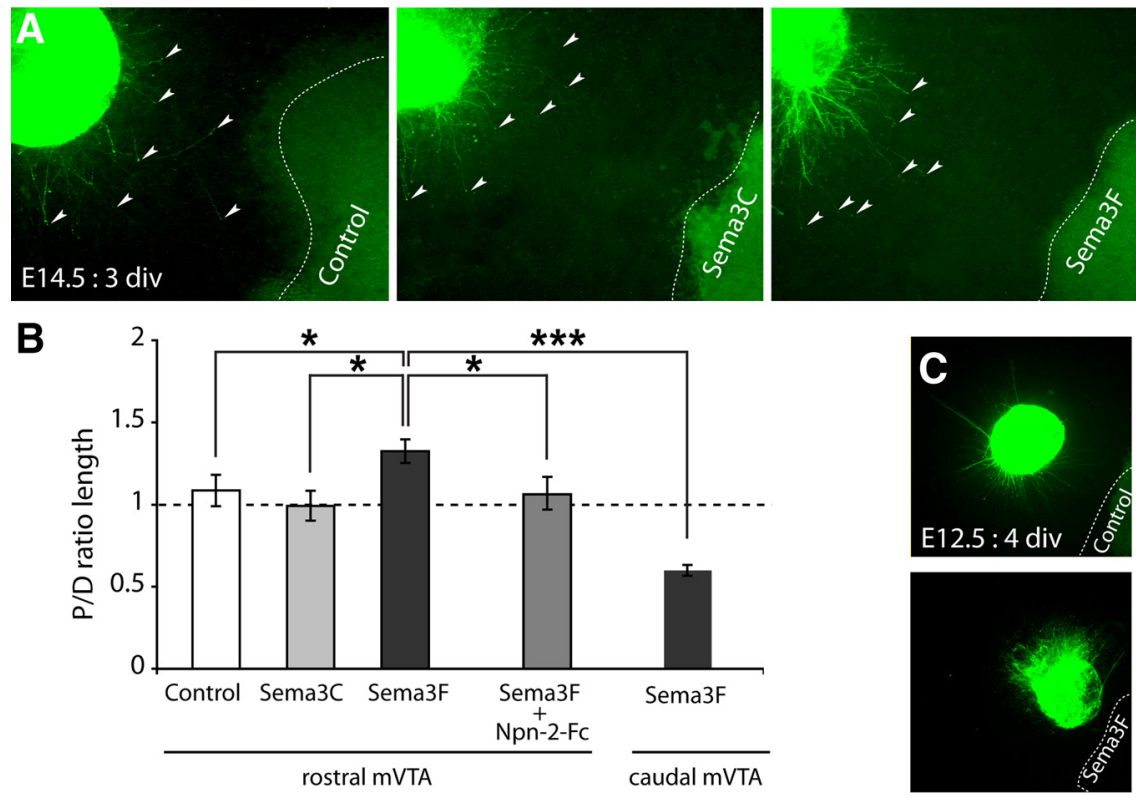

Figure 6. Sema3F is a bifunctional axon guidance cue for rostral mVTA axons. $\boldsymbol{A}$, TH immunostaining (green) of E14.5 rostral mVTA explants (proximal quadrants are shown; arrowheads indicate the leading edge of a set of axons) cultured adjacent to HEK293 cells (dotted line) secreting either control protein $(n=8)$, Sema3C $(n=10)$, or Sema3F $(n=11)$. B, Quantification of the length of TH-positive neurites in the proximal and distal quadrants of the culture assays shown in $A$. Graph shows average $P / D$ ratio \pm SEM. Note that TH-positive rostral mVTA axons are attracted by Sema3F (P/D ratio, 1.33; $p<0.05)$ but not by Sema3C (P/D ratio, 1.01) compared with control. This attraction is blocked by Npn-2-Fc ( $n=9 ; \mathrm{P} / \mathrm{D}$ ratio, 1.07; $p<0.05)$. Interestingly, TH-positive axons extending from caudal mVTA explants are repelled by Sema3F ( $n=12$; P/D ratio, $0.56 ; p<0.001)$. C, TH immunostaining (green) of E12.5 rostral mVTA explants cultured adjacent to HEK293 cells (dotted line) secreting either control protein $(n=12)$ or Sema3F ( $n=11)$. Axons extending from E12.5 rostral mVTA explants are repelled by Sema3F (P/D ratio, 0.52 ; $p<0.001)$. For each experimental condition, at least three independent experiments were performed. ${ }^{*} p<0.05 ;{ }^{* *} p<0.001$, one-way ANOVA. div, Days in vitro.
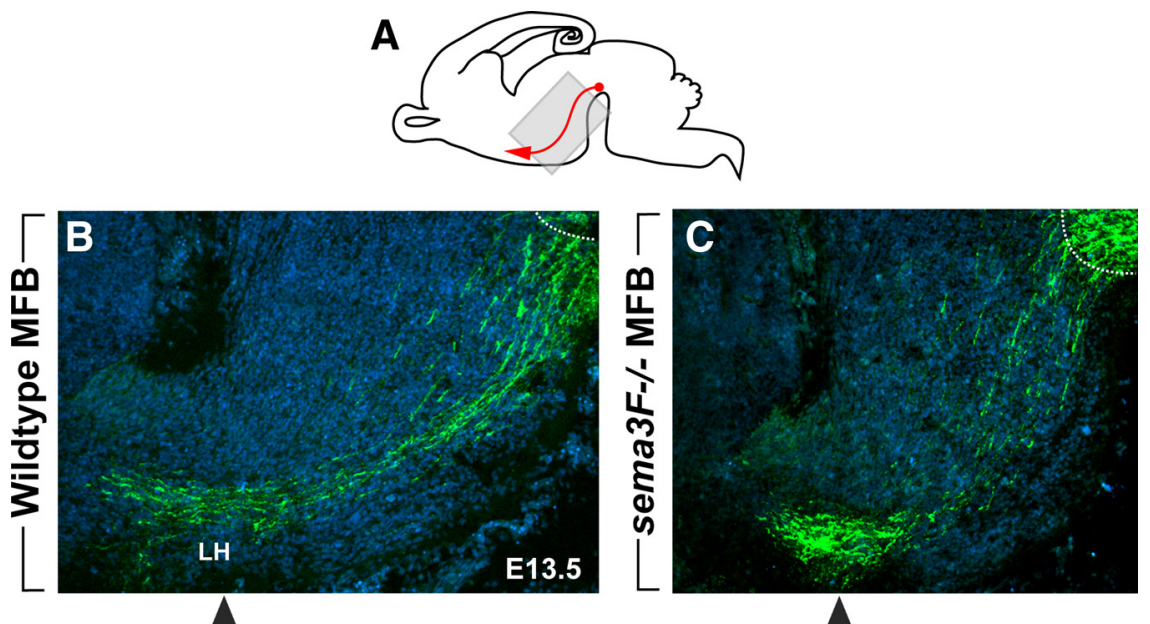

Figure 7. sema3F $F^{-1-}$ mice display defects in the rostral growth of $\mathrm{mdDA}$ axons in the forebrain. $\boldsymbol{A}$, Schematic showing the derivation of the sections shown in $\boldsymbol{B}$ and $\boldsymbol{C}$ in gray. The developing MFB is shown in red. $\boldsymbol{B}, \boldsymbol{C}$, Sagittal sections of E13.5 wt $(n=3)$ $(\boldsymbol{B})$ and sema3F ${ }^{-1-}(n=2)(\boldsymbol{C})$ mice are immunostained for TH (green) to visualize mdDA axons and counterstained with fluorescent Nissl (blue). The rostral extent of the mdDA neuron pool is indicated by a dotted line. $\boldsymbol{B}$, At E13.5, TH-positive axons project into the diencephalon and grow beyond the $\mathrm{LH}$ (arrowhead). $\mathbf{C}$, In sema3 $F^{-1-}$ mice, the MFB is defasciculated and spreads out over a larger area along the dorsoventral axis. In addition, in sema3 $F^{-1}$ - mice, TH-positive axons accumulate at the level of the LH and fail to extend further rostrally.

severely defasciculated and MFB width increased (supplemental Fig. S4, available at www.jneurosci.org as supplemental material). To determine the contribution of Npn-2 to Sema3F signaling in mdDA axon fasciculation and channeling in vivo, mdDA 

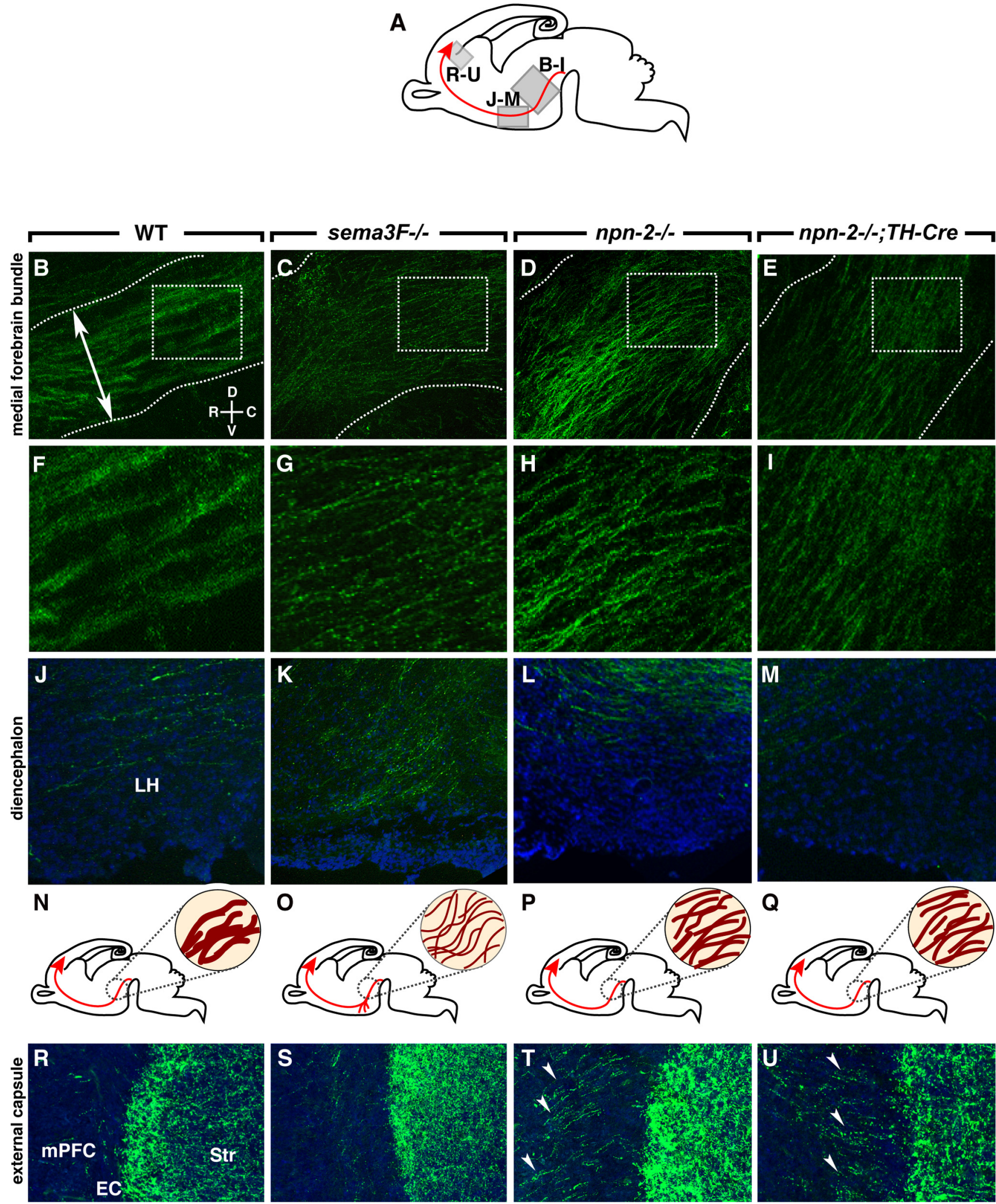

Figure 8. Abnormal channeling, fasciculation, rostral growth, and EC crossing of mdDA projections in sema3F- and npn-2-deficient mice. $A$, Schematic showing in gray the derivation of the sections in $\boldsymbol{B}-\boldsymbol{M}$ and $\boldsymbol{R}-\boldsymbol{U}$. The developing mesoprefrontal projection is shown in red. $\boldsymbol{B}-\boldsymbol{M}, \boldsymbol{R}-\boldsymbol{U}$, Sagittal sections at the level of the MFB $(\boldsymbol{B}-\boldsymbol{I})$, diencephalon $(\boldsymbol{J}-\boldsymbol{M})$, and EC $(\boldsymbol{R}-\boldsymbol{U})$ of E18.5 wt $(n=$

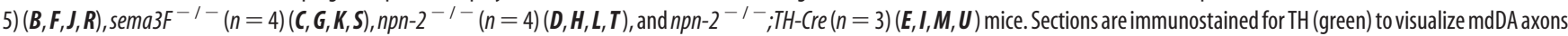
and counterstained with fluorescent Nissl (blue). The boxed areas in $\boldsymbol{B}-\boldsymbol{E}$ are enlarged in $\boldsymbol{F}-\mathbf{I} . \mathbf{N}-\mathbf{Q}$, Schematic representations of the development of the mesoprefrontal projection (in red) of wild-type $(\boldsymbol{N})$, sema3F $F^{-1-}(\mathbf{O}), n p n-2^{-1-}(\boldsymbol{P})$, and $n p n-2^{-1-} ; \mathrm{TH}$-Cre $(\boldsymbol{Q})$ mice with an enlargement at the level of the MFB (dark red within circle). $\boldsymbol{B}, \boldsymbol{F}$, In wild-type mice, the compact MFB (boundaries indicated by dotted lines) projects into the diencephalon with individual TH axons fasciculated into thick bundles. The double-arrowed line in $B$ indicates location of line used to quantify MFB width and axon fascicle number. $\boldsymbol{C}, \mathbf{G}$, In sema3F ${ }^{-1-}$ mutants, the MFB occupies a larger dorsoventral area and individual TH-positive axons are severely defasciculated. In $n p n-2^{-1-}(\boldsymbol{D}, \boldsymbol{H})$ and $n p n-2^{-I-} ;$ TH-Cre mice $(\boldsymbol{E}, I)$, the MFB is broader and TH-positive axons are defasciculated. In sema $3 F^{-I-}$ mutants $(\boldsymbol{K})$, but not in wild-type $(\boldsymbol{J})$, npn-2 $2^{-I-}($ Figure legend continues.) 
axon projections were analyzed in age-matched $n p n-2^{-1-}$ mice using TH immunohistochemistry (Giger et al., 2000). Inspection of E18.5 $n p n-2^{-1-}$ brains ( $n=4$; wild-type littermates, $n=3$ ) revealed that, although a few residual smaller axon fascicles were present in the MFB, the majority of TH-positive axons were defasciculated and the MFB was covering a larger dorsal-to-ventral area, as seen in sema3 $F^{-1-}$ mice (Fig. $8 D, H, P$ ). Quantification of the width of the MFB and of the number of thick axon fascicles within the MFB confirmed that the MFB in $s e m a 3 F^{-1-}$ and $n p n$ $2^{-1-}$ mice is significantly broader and contains fewer thick axon fascicles compared with control (MFB width: wt, $256.8 \pm 4.1 \mu \mathrm{m}$; sema3F ${ }^{-1-}, 362.3 \pm 12.8 \mu \mathrm{m}, p<0.0001 ; n p n-2^{-l-}, 363.1 \pm$ $3.4 \mu \mathrm{m}, p<0.0001$; axon fascicles: wt, $4.9 \pm 0.09$ fascicles; sema3F ${ }^{-l-}, 0.04 \pm 0,04$ fascicles, $p<0.0001 ; n p n-2^{-l-}, 0.18 \pm$ 0.07 fascicles, $p<0.0001)$. Together, these observations demonstrate a novel and indispensable role for Sema3F-Npn-2 signaling in the fasciculation and channeling of mdDA axons en route to their synaptic targets in the forebrain.

\section{sema3F-null mice exhibit defects in the rostral growth of mdDA axons in the diencephalon}

Another defect observed in E13.5 sema3 $\mathrm{F}^{-/-}$mice was the accumulation of TH-positive axons in the ventral diencephalon. Whereas many TH-positive axons had extended beyond the lateral hypothalamic $(\mathrm{LH})$ region at E13.5 in wild-type mice, most $\mathrm{TH}$-positive axons in E13.5 sema3 $\mathrm{F}^{-1-}$ mice accumulated in the LH failing to further project rostrally (Fig. $7 A-C$, black arrowhead). Although many TH-positive axons had managed to extend beyond the LH in sema3F $F^{-1-}$ mice by E18.5, numerous ectopic TH-positive axons remained detectable in the $\mathrm{LH}(n=4)$ (Fig. $8 \mathrm{~K}, \mathrm{O})$. None of the wild-type littermate controls $(n=4)$ showed ectopic axons within the ventral forebrain nor did E13.5 $(n=3)$ (data not shown) and E18.5 npn-2 $2^{-1-}$ mice $(n=3)$ (Fig. $8 J, L, N, P)$. Thus, Sema3F regulates the rostral growth of mdDA axons in the diencephalon in vivo through Npn-2-independent mechanisms. Interestingly, preliminary analysis of the innervation of $\mathrm{mPFC}$ and striatum by $\mathrm{TH}$-positive axons in adult sema $3 F^{-1-}$ mice shows that the majority of mdDA axons ultimately reaches their targets despite initial mistargeting in the ventral forebrain (data not shown).

\section{sema3F- and $n p n$-2-null mice show aberrant intracortical targeting of mesoprefrontal axons}

Mesoprefrontal mdDA axons follow two distinct trajectories to reach and innervate the $\mathrm{mPFC}$. Although the majority of axons innervate the mPFC by coursing dorsally just caudal of the $\mathrm{OB}$, a smaller subset of axons traverses the developing striatum and enters the mPFC by crossing the EC (Figs. 1, $8 R$ ). Interestingly, in $n p n-2^{-1-}$ but not sema3 $F^{-1-}$ mice, mdDA axons excessively crossed the EC and innervated the mPFC (Fig. $8 R-T$, arrowheads). Thus, Npn-2 controls the innervation of the mPFC by mdDA axons via the EC but does not rely on Sema3F for mediating this effect.

Mesoprefrontal mdDA axons reach the mPFC around E15 and accumulate within the SP before penetrating the overlaying

\footnotetext{
$\leftarrow$

(Figure legend continued.) (L), and npn-2 $2^{-1-} ; \mathrm{TH}$-Cre mice $(\boldsymbol{M})$, many axons in the diencephalon are displaced ventrally and appear to stall in the LH region. $\boldsymbol{R}-\boldsymbol{U}$, A subset of axons innervates the $\mathrm{mPFC}$ by crossing the EC $(\boldsymbol{R})$. Although this innervation appears normal in sema3 $\mathrm{F}^{-1-}$ mice $(\boldsymbol{S})$, TH-positive axons excessively cross the EC in npn-2 $2^{-1-}$ and npn-2 $2^{-1-}$; TH-Cremice (arrowheads). Str, Striatum.
}

$\mathrm{CP} \sim 2 \mathrm{~d}$ later (Fig. 2). During this period, Npn-2 is expressed in mesoprefrontal (rostral mVTA) neurons and their axons, and $s e m a 3 F$ is present in the CP of the mPFC. Furthermore, Npn-2 blocking tools neutralize the attractive effect of E14.5 mPFC on mesoprefrontal axons in vitro. Similarly, Sema3F can elicit rostral mVTA axon attraction through Npn-2. To evaluate the requirement for Sema3F and Npn-2 in the guidance of mesoprefrontal mdDA projections toward or within the developing $\mathrm{mPFC}$ in vivo, mdDA innervation of the $\mathrm{mPFC}$ was studied in E18.5 sema $3 F^{-1-}$ and $n p n-2^{-1-}$ mice using TH immunostaining.

Despite the pronounced defects in mdDA axon fasciculation, channeling, rostral growth, and EC crossing observed in sema $3 F^{-1-}$ and $n p n-2^{-1-}$ mice (Fig. 8), numerous TH-positive axons reached and innervated the $\mathrm{MPFC}$ in these mice at E18.5 (Fig. 9A-C,E-G). The targeting of TH-positive axons within the $\mathrm{mPFC}$ was, however, severely disrupted in both $\operatorname{sema} 3 \mathrm{~F}^{-1-}(n=$ $5)$ and $n p n-2^{-1-}(n=4)$ mice. Several distinct targeting defects were observed. First, the overall innervation of the mPFC was significantly reduced in sema $3 F^{-1-}$ mice compared with wildtype littermates (Fig. 9I, $p<0.001, J$ ), whereas $n p n-2^{-/-}$mice showed an increase in overall TH-positive axon density (Fig. 9I, $p<0.01$ compared with littermate control, $J$ ). Second, THpositive axons that normally coursed parallel to the pial surface within the compact structure of the SP, occupied a wider area that included the SP but expanded toward the ventricular zone in E18.5 sema3 $F^{-1-}$ but not $n p n-2^{-1-}$ mice $(9 A-C, E-G, J)$. Third, in $n p n-2^{-1-}$ mice but not sema3 $F^{-1-}$ mice, a large subset of TH-positive axons in the SP region displayed "club-like" endings (Fig. 9C, arrowheads). Fourth, the majority of TH-positive axons within the $\mathrm{CP}$ in $s e m a 3 F^{-1-}$ and $n p n-2^{-1-}$ mice did not project perpendicular to the pial surface, as observed in wild-type mice, but instead followed an aberrant trajectory diagonal or parallel to the pial membrane (Fig. $9 A-C, E-G$ ). To further assess this defect, the orientation of individual mdDA axons within the CP was visualized and quantified. In E18.5 wild-type mice, the majority of axons in the CP followed a trajectory perpendicular to the pial surface $\left(0-20^{\circ}\right)$, whereas axons in the CP of $s e m a 3 F^{-1-}$ and $n p n-2^{-I-}$ displayed a more random orientation (Fig. $9 E-G$, insets, $K$ ). These results suggest that Sema3F and Npn-2 are necessary to orient mesoprefrontal axons in the $\mathrm{CP}$ and identify a novel role for chemoattractive mechanisms in orienting afferent axonal projections in their target fields.

\section{Selective genetic ablation of $\boldsymbol{n p n - 2}$ in TH-positive neurons reveals a cell-autonomous requirement for $\mathrm{Npn}-2$ in mdDA axon guidance}

Close inspection of Nissl-stained sections of the mPFC of $s e m a 3 F^{-1-}$ and $n p n-2^{-1-}$ mice revealed the absence of a clear IZ/SP zone (data not shown) hinting at putative cortical neuron migration abnormalities. To assess this further, immunohistochemistry was performed for the presumptive cortical layer V/VI marker Tbr1 (Hevner et al., 2001). Intriguingly, in E18.5 sema3 $\mathrm{F}^{-1-}$ and $n p n-2^{-1-}$ mice, Tbr1-positive neurons were no longer confined to the CP but were also detected in more superficial and deeper cortical layers (supplemental Fig. $S 5 B-D$, available at www.jneurosci.org as supplemental material). Thus, Sema3F and Npn-2 are required for the migration of cortical neurons. This raised the question whether the mistargeting of mdDA axons in the mPFC in sema3 $F^{-1-}$ and $n p n-2^{-1-}$ mice is attributable to defects in axonal guidance or abnormal cortical neuron migration, or both. To discriminate between these two possible scenarios, we used a $\mathrm{TH}$-Cre BAC transgenic mouse line (Gong et al., 2007) in combination with a $n p n-2$ conditional mu- 

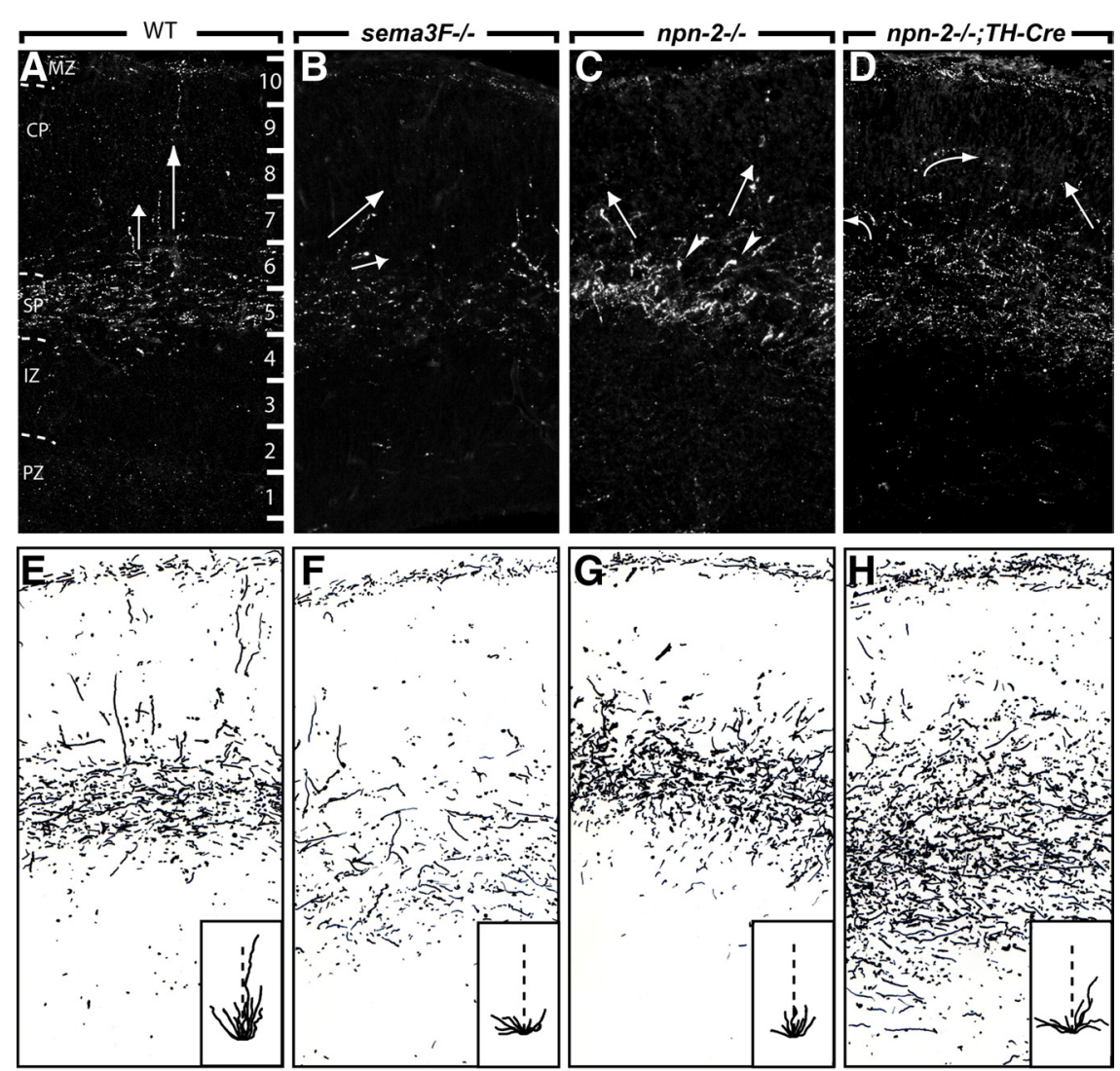

I
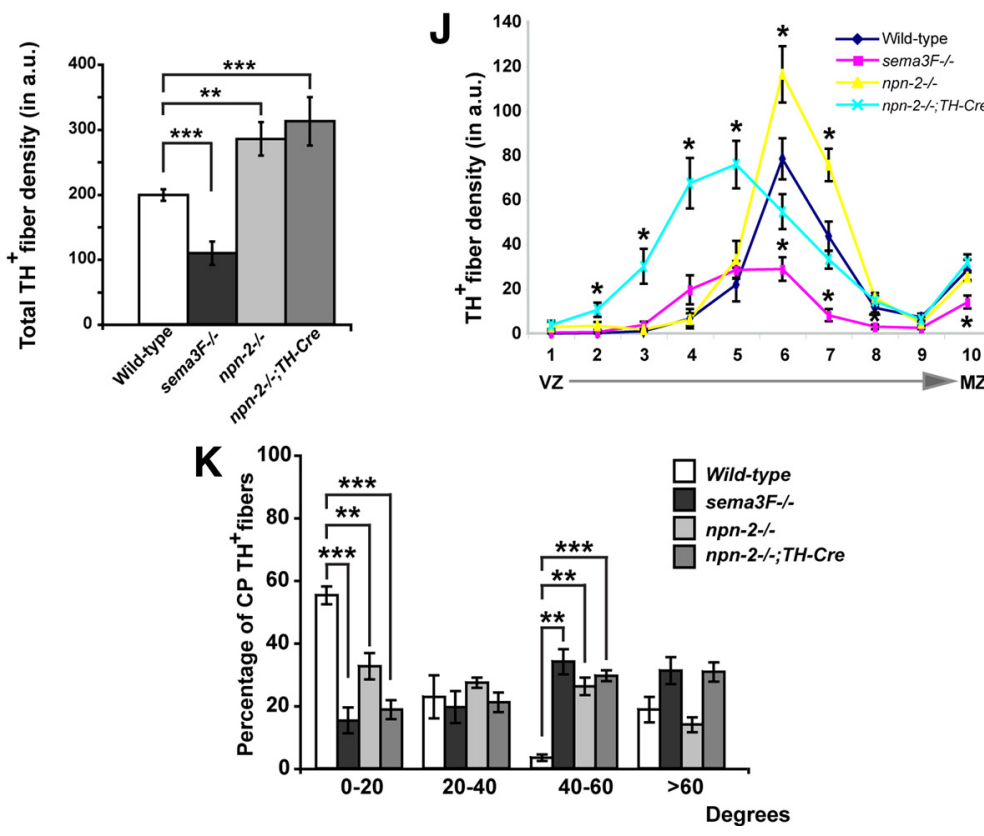

Figure 9. sema3F- and $n p n$-2-deficient mice display defects in intracortical targeting of mesoprefrontal axons. $\boldsymbol{A}-\boldsymbol{D}$, Sagittal sections of E18.5 mPFC in wild-type $(\boldsymbol{A})$, sema3F $F^{-1-}(\boldsymbol{B}), n p n-2^{-1-}(\boldsymbol{C})$, and $n p n-2^{-1-}$;TH-Cre mice (D) immunostained for $\mathrm{TH}$. The arrows indicate direction of growth of TH-positive axons in the CP. $\boldsymbol{B}-\boldsymbol{H}$, Camera lucida drawings of three consecutive sections within the mPFC of wild-type $(\boldsymbol{E})$, sema $3 F^{-1-}(\boldsymbol{F}), n p n-2^{-1-}(\boldsymbol{G})$, and $n p n-2^{-1-} ; \mathrm{TH}$-Cre mice $(\boldsymbol{H})$. The insets show axon orientation plots of randomly selected axons within the $C P$ [dotted line indicates trajectory perpendicular to the pial surface $\left(0^{\circ}\right)$ ]. $\boldsymbol{B}, \boldsymbol{F}$, Inset, Overall innervation of the $\mathrm{mPFC}$ in sema $3 \mathrm{~F}^{-/-}$mice is reduced and axons ectopically innervate deeper cortical areas. Furthermore, axons within the CP display a random orientation. $\mathbf{C}, \mathbf{G}$, Inset, In $n p n-2^{-/}$mutants, overall mPFC innervation is increased, mdDA axon growth in the $C P$ is randomized, and many axons form club-like structures (arrowheads). $\boldsymbol{D}, \boldsymbol{H}$, Inset In npn-2 $2^{-1-}$; TH-Cre mice, overall TH-positive innervation is increased and ectopic innervation of deeper cortical areas is prominent. TH-positive axon growth in the CP is randomized. I, Quantification of total TH axon density at E18.5 in the mPFC in arbitrary units (a.u.) confirms the qualitative observations shown in $\boldsymbol{A}-\boldsymbol{H}$. Graph shows average density \pm SEM. There is a significant decrease of TH-positive wiring in the mPFC of sema3F- $F^{-}$animals $(p<0.001)$ and an increase in $n p n-2^{-1-}$ and $n$ nn-2 $2^{-1-} ;$ TH-Cre animals ( $p<0.01$ and $p<0.05$, respectively) compared with wild type. J, Quantification of the distribution of tant mouse (Walz et al., 2002) to generate mice that lack $n p n-2$ exclusively in THpositive neurons. In mice that were heterozygous for the TH-Cre allele and either homozygous or heterozygous for the $n p n-2$ conditional allele $\left(n p n-2^{-1-} ; T H-\right.$ Cre or $\left.n p n-2^{+/-} ; \mathrm{TH}-\mathrm{Cre}\right)$, Cre recombination was limited to TH-positive neurons and their projections (data not shown). Furthermore, Nissl and Tbr1 immunostaining did not reveal cortical neuron migration defects in E18.5 $n p n-2^{-1-}$; TH-Cre mice $(n=3)$ (supplemental Fig. S5E, available at www.jneurosci.org as supplemental material). Analysis of $n p n$ $2^{-1-}$;TH-Cre mice $(n=3)$ using TH immunostaining revealed phenotypes similar to those seen in sema3F $F^{-1-}$ mice and/or $n p n-2^{-1-}$ mice. First, E18.5 npn$2^{-1-} ; \mathrm{TH}$-Cre mice displayed aberrant channeling and fasciculation of mdDA axons in the MFB (MFB width: $n p n-2^{-1-} ; \mathrm{TH}-$ Cre, $362.3 \pm 7.4 \mu \mathrm{m}, p<0.0001$; axon fascicles: $n p n-2^{-1-} ;$ TH-Cre, $0.22 \pm 0.05$ fascicles, $p<0.0001$ ) (Fig. 8E,I,Q). Second, as seen in $n p n-2^{-1-}$ mice, mdDA axons excessively crossed the EC in E18.5 npn-2 $2^{-1-} ; \mathrm{TH}-$ Cre mice (Fig. $8 U$, arrowheads). Third, overall innervation of the mPFC was increased in $n p n-2^{-I-} ;$ TH-Cre mice (Fig. 9I). Fourth, the area of mdDA innervation in the $\mathrm{mPFC}$ was expanded toward the ventricular zone in $n p n-$ $2^{-1-}$;TH-Cre mice, as seen in sema $3 F^{-1-}$ mice (Fig. 9D, $H, J$ ). Fifth, mdDA axons traversing the $\mathrm{CP}$ of the $\mathrm{mPFC}$ in $n p n-2^{-1-}$; $\mathrm{TH}$-Cre mice followed random trajectories instead of a normal radial path, as seen in age-matched $s e m a 3 F^{-1-}$ and $n p n-2^{-1-}$ mice (Fig. 9D, arrows; $H, K$ ). Finally, $n p n-2^{-1-} ;$ TH-Cre (and sema3F $F^{-1-}$ and $n p n-2^{-1-}$ mice) displayed caudally oriented mdDA axons at the MHB, as reported previously in another $n p n-2^{-1-}$ mouse strain (Yamauchi et al., 2009) (data not shown). None of these phenotypes were found in littermate controls $\left[n p n-2^{+1-} ; \mathrm{TH}-\right.$

TH-positive axon density in 10 bins dividing the MPFC as indicated in $\boldsymbol{A}$. Graph shows average density per bin \pm SEM. Note that innervation of the $\mathrm{mPFC}$ in sema $3 F^{-1-}$ and $n p n-2^{-1-} ;$ TH-Cre mice is shifted toward the lower bins. $\boldsymbol{K}$, Axon orientation histogram. Quantification of the orientation of mesoprefrontal axons in the $\mathrm{CP} . \mathrm{O}^{\circ}$ represents a trajectory perpendicular to the pial surface. Note that mesoprefrontal axon growth in the CP of the MPFC in all mutant mouse lines is random, whereas in wild-type mice most axons are biased toward a radial trajectory. White, Wild type; dark gray, sema3 $\mathrm{F}^{-1-}$; light gray, npn-2 $2^{-1-}$; intermediate gray, npn-2 ${ }^{-1-}$; TH-Cre. ${ }^{*} p<0.05 ;{ }^{* *} p<0.01 ;{ }^{* * *} p<$ 0.001 , one-way ANOVA. CP, Cortical plate; IZ, intermediate zone; MZ, marginal zone; SP, subplate; SVZ, subventricular zone; $\mathrm{VZ}$, ventricular zone. 
Cre mice $(n=3)$, npn-2 ${ }^{+/+} ;$TH-Cre mice $(n=3)$, and $n p n-2^{+/-} ;+$mice $\left.(n=3)\right]$. In all, these results indicate that Npn-2 is required cell autonomously in mdDA neurons to control mdDA axon channeling, fasciculation, EC crossing, and axon orientation in the $\mathrm{CP}$ of the $\mathrm{mPFC}$.

\section{Discussion}

Dopaminergic pathways mediate complex physiological functions and are a known therapeutic target, underscoring the need to define how these connections are established. The development of mdDA projections is remarkably complex and is contingent on the completion of several highly stereotypic events such as axon fasciculation, caudorostral extension, and targeting. The molecular programs regulating these disparate developmental processes in vivo remain mostly unknown. Here, we determine the embryonic origin and ontogeny of the mouse mesoprefrontal pathway and exploit these data to identify Sema3F as a bifunctional guidance cue and to demonstrate an unexpected requirement for Sema3F and its receptor Npn-2 in several fundamental and previously uncharacterized aspects of mdDA pathway development in vivo.

\section{Sema3F repulsion regulates fasciculation and channeling of mdDA axons}

Our results show that, in sema $3 F^{-1-}, n p n-$ $2^{-1-}$, and $n p n-2^{-1-} ; \mathrm{TH}-\mathrm{Cre}$ mice, axon bundles within the MFB are defasciculated and MFB width is increased. These results, coupled with sema3F expression surrounding the MFB (supplemental Fig. S6, available at www. jneurosci.org as supplemental material) and the ability of Sema3F to repel early mdDA axons in vitro (Hernández-Montiel et al., 2008; Yamauchi et al., 2009; present study), indicate that Sema3F-Npn-2-mediated surround repulsion is required in vivo for channeling and fasciculation of mdDA axons (Fig. 10). This is consistent with the role of Sema3s in other neuronal systems (Sahay et al., 2003; Walz et al., 2002; Falk et al., 2005). The only other guidance molecules implicated in MFB patterning to date are Slit proteins. Slits repel mdDA axons in vitro (Lin et al., 2005), and in Slit $1^{-1-}$;Slit2 $2^{-1-}$ mice, the MFB is split and displaced ventromedially (Bagri et al., 2002). Thus, whereas Slits control dorsolateral positioning of the MFB, Sema3F regulates its channeling and fasciculation.

\section{Sema3F enforces rostral growth of mdDA axons independent from Npn-2}

A characteristic feature of mdDA pathways is their extended longitudinal trajectory in the forebrain. The mechanisms that dictate axon growth along the rostrocaudal axis of the forebrain remain elusive. In sema $3 F^{-1-}$ mice, mdDA axons aberrantly accumulate in the LH. It is unlikely that this accumulation occurs simply because of abnormal channeling and fasciculation of mdDA axons, since it is not seen in $n p n-2^{-1-}$ mice. Instead, this axonal buildup, together with the repulsive effects of Sema3F and the presence of a, temporally expanding, high-caudal-to-low-rostral
sema3F gradient in the mesodiencephalon (supplemental Fig. S6, available at www.jneurosci.org as supplemental material) supports the idea that graded Sema3F expression dictates the rostral growth of mdDA projections within the forebrain (Fig. 10). Similar "push" mechanisms have been attributed to SHH (sonic hedgehog) and Wnt-5a in the growth of spinal pathways (Bourikas et al., 2005; Liu et al., 2005). mdDA axons in E13.5 sema $3 F^{-1-}$ mice do not simply display delayed rostral growth, but rather specifically accumulate in the LH. A possible explanation for this observation supported by the reported chemoattractive properties of cultured rat MFB (Gates et al., 2004) is that the loss of Sema3F unmasks a chemoattractive activity in the LH. Netrin-1 is enriched in the LH (supplemental Fig. S6, available at www.jneurosci.org as supplemental material) (Deiner and Sretavan, 1999) and positively influences mdDA guidance in vitro (Lin et al., 2005). Together, these observations hint at a model in which the LH expresses netrin-1, or another chemoattractant, to first attract mdDA axons rostrally, after which Sema3F dominates this attractive force and repels mdDA axons passed the LH. Future experiments will address these and other possibilities.

\section{Axon attraction by Sema3F controls the intracortical targeting of mdDA axons}

After a waiting period in the SP, mdDA axons innervate the overlaying CP following a trajectory perpendicular to the pial surface (Fig. 10). This directed growth suggests that mdDA axons in the $\mathrm{CP}$ might be guided by extracellular signals. Our experiments identify Sema3F as such an axon orientation signal. We find 
Npn-2 expression in mesoprefrontal neurons/axons and show that blocking Npn-2 neutralizes the attractive effect of mPFC on mesoprefrontal axons in vitro. Sema3F mediates this attractive signal since it is expressed in the CP and, by analogy to the mPFC, can elicit rostral mVTA axon attraction through Npn-2 in vitro. Finally, mdDA axons enter the CP of the mPFC in $s e m a 3 F^{-1-}$, $n p n-2^{-1-}$, and $n p n-2^{-1-} ; \mathrm{TH}-\mathrm{Cre}$ mice but are randomly oriented. Thus, axon attraction by Sema3F through Npn-2 appears to be necessary for correct orientation of mdDA axons in the CP in vivo (Fig. 10). In all, our findings demonstrate that regulation of axon orientation in the target field occurs by chemoattractive mechanisms.

Although sema3F is expressed in the E14.5 CP and attracts mesoprefrontal axons as early as $\sim \mathrm{E} 15.5$, these axons do not enter the $\mathrm{CP}$ until $\sim \mathrm{E} 17.5$. One model to explain this behavior is that the $\mathrm{CP}$ contains nonpermissive molecules such as semaphorins, Slits, and ephrins (Skaliora et al., 1998; Mackarehtschian et al., 1999; Marillat et al., 2002) that dominate Sema3F attraction until $\sim$ E17.5. Alternatively, the SP may be much more permissive for mesoprefrontal axons than the CP before E17.5, as has been shown for migrating GABAergic interneurons (LópezBendito et al., 2008). This latter model is supported by the ability of early mPFC to attract early rostral mVTA axons in an Npn-2independent manner (supplemental Fig. S7, available at www. jneurosci.org as supplemental material). Additional studies will determine the precise mechanisms that regulate the intracortical waiting period of mesoprefrontal axons. Furthermore, since the intracortical behavior of mdDA axons resembles that of other Npn-positive cortical afferents/migrating interneurons (LópezBendito and Molnár, 2003; López-Bendito et al., 2008), it will be interesting to explore whether the intracortical targeting principles determined here also apply to those systems.

\section{Npn-2 controls mPFC innervation through the external capsule}

Sema3F- and npn-2-deficient mice exhibit identical defects in $\mathrm{CP}$ mdDA axon orientation. Other cortical phenotypes are, however, not shared among all three genetic models. The interpretation of these phenotypes is, at least in $s e m a 3 F^{-1-}$ and $n p n-2^{-1-}$ mice, complicated by concurrent cortical neuron migration defects (supplemental Fig. S5, available at www.jneurosci.org as supplemental material). Nevertheless analysis of $n p n-2^{-1-} ; \mathrm{TH}$-Cre mice reveals a Npn-2-dependent effect that may not rely on Sema3F. During development, mdDA axons reach the mPFC following two different routes, one of which is through the striatum and EC (Fig. 8R). Interestingly, $n p n-2^{-1-} ; \mathrm{TH}$-Cre mice (and $n p n-2^{-1-}$ mice) display an increase in the number of mdDA axons that enter the mPFC via the EC. This defect is not seen in $s e m a 3 F^{-1-}$ mice, and sema3C but not sema3F is highly expressed in cortical layers lining the EC (Bagnard et al., 1998; present study). Although Sema3C is an attractant for early rat mdDA axons (Hernández-Montiel et al., 2008), its effect on mesostriatal axons during later stages is most likely repulsive in nature (Yamauchi et al., 2009). This suggests that Sema3C may regulate the innervation of the $\mathrm{mPFC}$ by mdDA axons through the EC (Fig. 10).

\section{Sema3F is a bifunctional guidance cue for mdDA axons}

Our results identify Sema3F as a bifunctional guidance cue that, by analogy to other Sema3s (Falk et al., 2005; Chauvet et al., 2007), can exert both axon-repulsive and -attractive effects. Remarkably, rostral, but not caudal, mdDA axons change their response to Sema3F as they develop (from repulsion to attraction).
Previous work demonstrates that retinal and commissural axons acquire repulsive semaphorin responsiveness in an agedependent manner (Zou et al., 2000; Campbell et al., 2001). Our findings reveal an additional regulatory mechanism of semaphorin responsiveness (i.e., a stage-dependent switch from one chemotropic response to another). How is this change regulated? A plausible mechanism, supported by observations in other neural systems (for review, see Chao et al., 2009), is that signals along the mesoprefrontal trajectory trigger changes in axon responsiveness to Sema3F. Interestingly, the change in Sema3F responses of mesoprefrontal axons coincides with their arrival in the SP, a well known intermediate target (Allendoerfer and Shatz, 1994; LópezBendito and Molnár, 2003). It is therefore tempting to speculate that the SP releases unidentified molecular cues that regulate the response of mesoprefrontal axons to Sema3F. Although additional experiments are required to identify these cues, they might include extracellular matrix (ECM) components. The SP is rich in ECM components (Henke-Fahle et al., 1996; Voss et al., 2008), which can modulate axonal responses to guidance cues (Höpker et al., 1999; Nguyen-Ba-Charvet et al., 2001; Kantor et al., 2004).

The mechanisms underlying axon attraction mediated by Sema3s are poorly understood (Zhou et al., 2008). Our work shows a requirement for Npn-2 in both Sema3F axon attraction and repulsion. In addition to Npns, plexins, IgCAMs (Igsuperfamily cell adhesion molecules), cyclic nucleotides, and cytosolic kinases have been implicated in Sema3 axon attraction (Song et al., 1998; Castellani et al., 2000; Falk et al., 2005; Chauvet et al., 2007; Ding et al., 2007). The combination of extrinsic and intrinsic factors that is required to confer mdDA neuron responsiveness to the attractive properties of Sema3F remains to be determined.

\section{Concluding remarks}

Here, we show that Sema3F is a bifunctional guidance cue for mdDA axons, some of which uniquely regulate their responsiveness to Sema3F as they develop. Sema3F repulsion controls previously uncharacterized aspects of mdDA pathway development through both Npn-2-dependent (fasciculation, channeling) and Npn-2-independent (rostral growth) mechanisms. Unexpectedly, axon attraction by Sema3F and Npn-2 is required to orient mdDA axons in the $\mathrm{CP}$, defining a novel role for chemoattraction in determining axon orientation in the target field.

\section{References}

Allendoerfer KL, Shatz CJ (1994) The subplate, a transient neocortical structure: its role in the development of connections between thalamus and cortex. Annu Rev Neurosci 17:185-218.

Anderson CN, Ohta K, Quick MM, Fleming A, Keynes R, Tannahill D (2003) Molecular analysis of axon repulsion by the notochord. Development 130:1123-1133.

Bagnard D, Lohrum M, Uziel D, Püschel AW, Bolz J (1998) Semaphorins act as attractive and repulsive guidance signals during the development of cortical projections. Development 125:5043-5053.

Bagri A, Marín O, Plump AS, Mak J, Pleasure SJ, Rubenstein JL, TessierLavigne M (2002) Slit proteins prevent midline crossing and determine the dorsoventral position of major axonal pathways in the mammalian forebrain. Neuron 33:233-248.

Björklund A, Dunnett SB (2007) Dopamine neuron systems in the brain: an update. Trends Neurosci 30:194-202.

Bourikas D, Pekarik V, Baeriswyl T, Grunditz A, Sadhu R, Nardó M, Stoeckli ET (2005) Sonic hedgehog guides commissural axons along the longitudinal axis of the spinal cord. Nat Neurosci 8:297-304.

Campbell DS, Regan AG, Lopez JS, Tannahill D, Harris WA, Holt CE (2001) Semaphorin 3A elicits stage-dependent collapse, turning, and branching in Xenopus retinal growth cones. J Neurosci 21:8538-8547.

Castellani V, Chédotal A, Schachner M, Faivre-Sarrailh C, Rougon G (2000) 
Analysis of the L1-deficient mouse phenotype reveals cross-talk between Sema3A and L1 signaling pathways in axonal guidance. Neuron 27:237-249.

Chao DL, Ma L, Shen K (2009) Transient cell-cell interactions in neural circuit formation. Nat Rev Neurosci 10:262-271.

Chauvet S, Cohen S, Yoshida Y, Fekrane L, Livet J, Gayet O, Segu L, Buhot MC, Jessell TM, Henderson CE, Mann F (2007) Gating of Sema3E/PlexinD1 signaling by neuropilin-1 switches axonal repulsion to attraction during brain development. Neuron 56:807-822.

Chen H, Chédotal A, He Z, Goodman CS, Tessier-Lavigne M (1997) Neuropilin-2, a novel member of the neuropilin family, is a high affinity receptor for the semaphorins Sema E and Sema IV but not Sema III. Neuron 19:547-559.

Cooper MA, Kobayashi K, Zhou R (2009) Ephrin-A5 regulates the formation of the ascending midbrain dopaminergic pathways. Dev Neurobiol 69:36-46.

Deiner MS, Sretavan DW (1999) Altered midline axon pathways and ectopic neurons in the developing hypothalamus of netrin-1- and DCCdeficient mice. J Neurosci 19:9900-9912.

Dickson BJ (2002) Molecular mechanisms of axon guidance. Science 298:1959-1964.

Ding S, Luo JH, Yuan XB (2007) Semaphorin-3F attracts the growth cone of cerebellar granule cells through cGMP signaling pathway. Biochem Biophys Res Commun 356:857-863.

Falk J, Julien F, Bechara A, Fiore R, Nawabi H, Zhou H, Hoyo-Becerra C, Bozon M, Rougon G, Grumet M, Püschel AW, Sanes JR, Castellani V (2005) Dual functional activity of semaphorin 3B is required for positioning the anterior commissure. Neuron 48:63-75.

Fallon JH (1981) Collateralization of monoamine neurons: mesotelencephalic dopamine projections to caudate, septum, and frontal cortex. J Neurosci 1:1361-1368.

Funato H, Saito-Nakazato Y, Takahashi H (2000) Axonal growth from the habenular nucleus along the neuromere boundary region of the diencephalon is regulated by semaphorin $3 \mathrm{~F}$ and netrin-1. Mol Cell Neurosci 16:206-220.

Gates MA, Coupe VM, Torres EM, Fricker-Gates RA, Dunnett SB (2004) Spatially and temporally restricted chemoattractive and chemorepulsive cues direct the formation of the nigro-striatal circuit. Eur J Neurosci 19:831-844.

Giger RJ, Wolfer DP, De Wit GM, Verhaagen J (1996) Anatomy of rat semaphoring III/collapsing-1 mRNA expression and relationship to developing nerve tracts during neuroembryogenesis. J Comp Neurol 375:378-392.

Giger RJ, Urquhart ER, Gillespie SK, Levengood DV, Ginty DD, Kolodkin AL (1998) Neuropilin-2 is a receptor for semaphorin IV: insight into the structural basis of receptor function and specificity. Neuron 21:1079-1092.

Giger RJ, Cloutier JF, Sahay A, Prinjha RK, Levengood DV, Moore SE, Pickering S, Simmons D, Rastan S, Walsh FS, Kolodkin AL, Ginty DD, Geppert M (2000) Neuropilin-2 is required in vivo for selective axon guidance responses to secreted semaphorins. Neuron 25:29-41.

Gong S, Doughty M, Harbaugh CR, Cummins A, Hatten ME, Heintz N, Gerfen CR (2007) Targeting Cre recombinase to specific neuron populations with bacterial artificial chromosome constructs. J Neurosci 27:9817-9823.

Halladay AK, Tessarollo L, Zhou R, Wagner GC (2004) Neurochemical and behavioral deficits consequent to expression of a dominant negative EphA5 receptor. Brain Res Mol Brain Res 123:104-111.

Henke-Fahle S, Mann F, Götz M, Wild K, Bolz J (1996) Dual action of a carbohydrate epitope on afferent and efferent axons in cortical development. J Neurosci 16:4195-4206.

Hernández-Montiel HL, Tamariz E, Sandoval-Minero MT, VarelaEchavarría A (2008) Semaphorins 3A, 3C, and 3F in mesencephalic dopaminergic axon pathfinding. J Comp Neurol 506:387-397.

Hevner RF, Shi L, Justice N, Hsueh Y, Sheng M, Smiga S, Bulfone A, Goffinet AM, Campagnoni AT, Rubenstein JL (2001) Tbr1 regulates differentiation of the preplate and layer 6. Neuron 29:353-366.

Höpker VH, Shewan D, Tessier-Lavigne M, Poo M, Holt C (1999) Growthcone attraction to netrin- 1 is converted to repulsion by laminin-1. Nature 401:69-73.

Huber AB, Kolodkin AL, Ginty DD, Cloutier JF (2003) Signaling at the growth cone: ligand-receptor complexes and the control of axon growth and guidance. Annu Rev Neurosci 26:509-563.
Ikemoto S (2007) Dopamine reward circuitry: two projection systems from the ventral midbrain to the nucleus accumbens-olfactory tubercle complex. Brain Res Rev 56:27-78.

Ito K, Kawasaki T, Takashima S, Matsuda I, Aiba A, Hirata T (2008) Semaphorin $3 \mathrm{~F}$ confines ventral tangential migration of lateral olfactory tract neurons onto the telencephalon surface. J Neurosci 28:4414-4422.

Jacobowitz DM, Abott LC (1998) Chemoarchitectonic atlas of the developing mouse brain. Boca Raton, FL: CRC.

Kalsbeek A, Voorn P, Buijs RM, Pool CW, Uylings HBM (1988) Development of the dopaminergic innervation of the prefrontal cortex of the rat. J Comp Neurol 269:58-72.

Kantor DB, Chivatakarn O, Peer KL, Oster SF, Inatani M, Hansen MJ, Flanagan JG, Yamaguchi Y, Sretavan DW, Giger RJ, Kolodkin AL (2004) Semaphorin $5 \mathrm{~A}$ is a bifunctional axon guidance cue regulated by heparan and chondroitin sulfate proteoglycans. Neuron 44:961-975.

Kolk SM, Whitman MC, Yun ME, Shete P, Donoghue MJ (2006) A unique subpopulation of Tbr1-expressing deep layer neurons in the developing cerebral cortex. Mol Cell Neurosci 32:200-214.

Kolodkin AL, Levengood DV, Rowe EG, Tai YT, Giger RJ, Ginty DD (1997) Neuropilin is a semaphorin III receptor. Cell 90:753-762.

Kusy S, Funkelstein L, Bourgais D, Drabkin H, Rougon G, Roche J, Castellani V (2003) Redundant functions but temporal and regional regulation of two alternatively spliced isoforms of semaphorin $3 \mathrm{~F}$ in the nervous system. Mol Cell Neurosci 24:409-418.

Lammel S, Hetzel A, Häckel O, Jones I, Liss B, Roeper J (2008) Unique properties of mesoprefrontal neurons within a dual mesocorticolimbic dopamine system. Neuron 57:760-773.

Lin L, Rao Y, Isacson O (2005) Netrin-1 and slit-2 regulate and direct neurite growth of ventral midbrain dopaminergic neurons. Mol Cell Neurosci 28:547-555.

Liu Y, Shi J, Lu CC, Wang ZB, Lyuksyutova AI, Song XJ, Song X, Zou Y (2005) Ryk-mediated Wnt repulsion regulates posterior-directed growth of corticospinal tract. Nat Neurosci 8:1151-1159.

López-Bendito G, Molnár Z (2003) Thalamocortical development: how are we going to get there? Nat Rev Neurosci 4:276-289.

López-Bendito G, Sánchez-Alcañiz JA, Pla R, Borrell V, Picó E, Valdeolmillos M, Marín O (2008) Chemokine signaling controls intracortical migration and final distribution of GABAergic interneurons. J Neurosci 28:1613-1624.

Lyuksyutova AI, Lu CC, Milanesio N, King LA, Guo N, Wang Y, Nathans J, Tessier-Lavigne M, Zou Y (2003) Anterior-posterior guidance of commissural axons by Wnt-frizzled signaling. Science 302:1984-1988.

Mackarehtschian K, Lau CK, Caras I, McConnell SK (1999) Regional differences in the developing cerebral cortex revealed by ephrin-A5 expression. Cereb Cortex 9:601-610.

Margolis EB, Lock H, Chefer VI, Shippenberg TS, Hjelmstad GO, Fields HL (2006) Kappa opioids selectively control dopaminergic neurons projecting to the prefrontal cortex. Proc Natl Acad Sci U S A 103:2938-2942.

Marillat V, Cases O, Nguyen-Ba-Charvet KT, Tessier-Lavigne M, Sotelo C, Chédotal A (2002) Spatiotemporal expression patterns of slit and robo genes in the rat brain. J Comp Neurol 442:130-155.

Marín O, Yaron A, Bagri A, Tessier-Lavigne M, Rubenstein JL (2001) Sorting of striatal and cortical interneurons regulated by semaphorinneuropilin interactions. Science 293:872-875.

Nakamura S, Ito Y, Shirasaki R, Murakami F (2000) Local directional cues control growth polarity of dopaminergic axons along the rostrocaudal axis. J Neurosci 20:4112-4119.

Nguyen-Ba-Charvet KT, Brose K, Marillat V, Sotelo C, Tessier-Lavigne M, Chédotal A (2001) Sensory axon response to substrate-bound Slit2 is modulated by laminin and cyclic GMP. Mol Cell Neurosci 17:1048-1058.

Osborne NJ, Begbie J, Chilton JK, Schmidt H, Eickholt BJ (2005) Semaphorin/neuropilin signaling influences the positioning of migratory neural crest cells within the hindbrain region of the chick. Dev Dyn 232:939-949.

Pascual M, Pozas E, Soriano E (2005) Role of class 3 semaphorins in the development and maturation of the septohippocampal pathway. Hippocampus 15:184-202.

Pasterkamp RJ, Peschon JJ, Spriggs MK, Kolodkin AL (2003) Semaphorin 7A promotes axon outgrowth through integrins and MAPKs. Nature 424:398-405.

Pasterkamp RJ, Kolk SM, Hellemons AJ, Kolodkin AL (2007) Expression patterns of semaphorin7A and plexinC1 during rat neural development 
suggest roles in axon guidance and neuronal migration. BMC Dev Biol 7:98.

Petros TJ, Rebsam A, Mason CA (2008) Retinal axon growth at the optic chiasm: to cross or not to cross. Annu Rev Neurosci 31:295-315.

Sahay A, Molliver ME, Ginty DD, Kolodkin AL (2003) Semaphorin 3F is critical for development of limbic system circuitry and is required in neurons for selective CNS axon guidance events. J Neurosci 23:66716680.

Savitt JM, Dawson VL, Dawson TM (2006) Diagnosis and treatment of Parkinson disease: molecules to medicine. J Clin Invest 116:1744-1754.

Schambra UB, Lauder JM, Silver J (1992) Atlas of the prenatal mouse brain. San Diego: Academic.

Sieber BA, Kuzmin A, Canals JM, Danielsson A, Paratcha G, Arenas E, Alberch J, Ogren SO, Ibáñez CF (2004) Disruption of EphA/ephrin-a signaling in the nigrostriatal system reduces dopaminergic innervation and dissociates behavioral responses to amphetamine and cocaine. Mol Cell Neurosci 26:418-428.

Skaliora I, Singer W, Betz H, Püschel AW (1998) Differential patterns of semaphorin expression in the developing rat brain. Eur J Neurosci 10:1215-1229.

Smidt MP, Burbach JP (2007) How to make a mesodiencephalic dopaminergic neuron. Nat Rev Neurosci 8:21-32.

Song H, Ming G, He Z, Lehmann M, McKerracher L, Tessier-Lavigne M, Poo M (1998) Conversion of neuronal growth cone responses from repulsion to attraction by cyclic nucleotides. Science 281:1515-1518.

Swanson LW (1982) The projections of the ventral tegmental area and adjacent regions: a combined fluorescent retrograde tracer and immunofluorescence study in the rat. Brain Res Bull 9:321-353.

Tamamaki N, Fujimori K, Nojyo Y, Kaneko T, Takauji R (2003) Evidence that Sema3A and Sema3F regulate the migration of GABAergic neurons in the developing neocortex. J Comp Neurol 455:238-248.
Tessier-Lavigne M, Goodman CS (1996) The molecular biology of axon guidance. Science 274:1123-1133.

Thompson L, Barraud P, Andersson E, Kirik D, Björklund A (2005) Identification of dopaminergic neurons of nigral and ventral tegmental area subtypes in grafts of fetal ventral mesencephalon based on cell morphology, protein expression, and efferent projections. J Neurosci 25:64676477.

Van den Heuvel DM, Pasterkamp RJ (2008) Getting connected in the dopamine system. Prog Neurobiol 85:75-93.

Verney C (1999) Distribution of the catecholaminergic neurons in the central nervous system of human embryos and fetuses. Microsc Res Tech 46:24-47.

Voss AK, Britto JM, Dixon MP, Sheikh BN, Collin C, Tan SS, Thomas T (2008) C3G regulates cortical neuron migration, preplate splitting and radial glial cell attachment. Development 135:2139-2149.

Walz A, Rodriguez I, Mombaerts P (2002) Aberrant sensory innervation of the olfactory bulb in neuropilin-2 mutant mice. J Neurosci 22:40254035.

Winterer G, Weinberger DR (2004) Genes, dopamine and cortical signalto-noise ratio in schizophrenia. Trends Neurosci 27:683-690.

Yamauchi K, Mizushima S, Tamada A, Yamamoto N, Takashima S, Murakami F (2009) FGF8 signaling regulates growth of midbrain dopaminergic axons by inducing semaphorin 3F. J Neurosci 29:4044-4055.

Yue Y, Widmer DA, Halladay AK, Cerretti DP, Wagner GC, Dreyer JL, Zhou R (1999) Specification of distinct dopaminergic neural pathways: roles of the Eph family receptor EphB1 and ligand ephrin-B2. J Neurosci 19:2090-2101.

Zhou Y, Gunput RA, Pasterkamp RJ (2008) Semaphorin signaling: progress made and promises ahead. Trends Biochem Sci 33:161-170.

Zou Y, Stoeckli E, Chen H, Tessier-Lavigne M (2000) Squeezing axons out of the gray matter: a role for slit and semaphorin proteins from midline and ventral spinal cord. Cell 102:363-375. 